\title{
Marginal Beta Limit For Neoclassical Tearing Modes In JET H-Mode Discharges
}

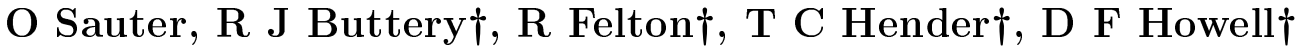 \\ and contributors to the EFDA-JET Workprogramme $\dagger \uparrow$
}

Centre de Recherches en Physique des Plasmas, EPFL, Association

EURATOM-Confédération Suisse, PPB-Ecublens, 1015 Lausanne, Switzerland

$\dagger$ EURATOM/UKAEA Fusion Association, Culham Science Centre, Abingdon, Oxon

OX14 3DB, UK

††See annex of J. Pamela et al, "Overview of Recent JET Results and Future

Perspectives", Fusion Energy 2000 (Proc. 18th Int. Conf. Sorrento, 2000), IAEA,

Vienna (2001)

\begin{abstract}
A Neoclassical tearing mode (NTM) requires a finite size seed island to become unstable. Usually the local pressure gradient is relatively large at the beta values needed for these seed islands to destabilize the NTMs. Therefore the island has a large growth rate at mode onset and grows rapidly to its saturated island width. This width is proportional to beta as long as it is well above the marginal beta limit below which the mode is stable. The marginal beta limit is independent of the seed island trigger mechanism and provides detailed information on the stabilising terms in the modified Rutherford equation, which are not unambiguously determined theoretically. It is shown that in JET the marginal normalised beta limit for the $3 / 2$ mode, $\beta_{N, \text { marg }}$, is of the order of 0.5 to 1 for magnetic fields between $3.3 \mathrm{~T}$ and $1 \mathrm{~T}$, with $q_{95} \approx 3.3$, and near the H-L transition. Therefore all H-modes with typical q profiles $\left(q_{95} \approx 3.3\right)$ are metastable in JET to $3 / 2$ NTMs. In addition the marginal island width is of the order of $2-4 \mathrm{~cm}$ and the stabilising terms are such that they influence the saturated island width when it is smaller than $4-6 \mathrm{~cm}$ in these H-mode discharges. It is also shown that detailed analyses of the time evolution of the island width with slow beta ramp-down suggest that the convective form of the stabilisation term due to the " $\chi_{\perp}$ model" is more appropriate and can explain the island decay between $4-6 \mathrm{~cm}$ to the marginal island width, while the polarisation current model can explain the rapid stabilisation when $\beta<\beta_{\text {marg. }}$. The range of values of the different stabilising terms are discussed in detail. In particular it is shown that the mode is stabilised and has a large negative growth rate, when the effect of the stabilising terms is such as to reduce the saturated width by a factor of two.
\end{abstract}

Submitted to: Plasma Phys. Control. Fusion

E-mail: olivier.sauter@epfl.ch 


\section{Introduction}

It is now well established that neoclassical tearing modes (NTMs), driven by the perturbed bootstrap current, can be destabilised at values of $\beta$, the ratio of plasma pressure $p$ to magnetic pressure $B_{0}^{2} / 2 \mu_{0}$, much lower than the ideal $\beta$ limit $[1,2,3,4,5,6]$. These modes have been observed in most tokamaks in standard discharges (monotonic safety factor profiles, q) with the high confinement mode (H-mode). It has been shown that the effect on confinement is well estimated by assuming a flat pressure profile across the island and therefore leads to $10-20 \%$ confinement degradation for $m=3 / n=2$ NTMs and about twice that for $2 / 1$ modes (where $m, n$ are the poloidal and toroidal mode numbers respectively). A maximum confinement degradation of about $20 \%$ (depending on plasma current) is acceptable in the predicted standard mode of operation in ITER-FEAT [7], in order to fulfill its main goal. Therefore it is important to better understand the physics and dependencies of the main terms involved in the evolution of the saturated island width.

In recent years, the main experimental studies have concentrated on the characterisation of the plasma parameters at the onset of the mode, and in particular on the dependence of $\beta_{N}$ or $\beta_{p}$ on $\rho *$ and $\nu_{i i} *[5,8,3,9]$, where $\beta_{N}$ is the normalised $\beta\left(100 \beta /\left(I_{p} / a B_{0}\right)\right), \beta_{p}$ the poloidal beta, $I_{p}$ the plasma current $[\mathrm{MA}]$, a the minor radius $[\mathrm{m}], B_{0}$ the vacuum magnetic field $[\mathrm{T}]$ at the plasma major radius, $\rho *$ the normalised ion Larmor radius and $\nu_{i i} *=\nu_{i i} / \epsilon \omega_{e} *$ a normalised ion collisionality related to the polarisation model [10], with $\epsilon$ the inverse aspect ratio and $\omega_{e} *$ the electron diamagnetic frequency. These studies give insights on the NTM mode formation and on the comparison between different size tokamaks. However, due to stabilising terms, the NTMs need a seed island large enough such that the perturbed bootstrap current can drive the island further. Therefore the studies at mode onset depend strongly on the seed island size and formation mechanisms $[8,11]$. In particular it is known that the values of $\beta$ at mode onset are generally much larger than the marginal $\beta$ limit, $\beta_{\text {marg }}$, below which NTMs are unconditionally stable [2]. The difference between the two depends on the seed islands available in the discharge. It has been recently shown in the Joint European Torus (JET) that by inducing a long sawtooth period, NTMs can be destabilised at much lower $\beta$, close to $\beta_{\text {marg }}[12]$. Therefore new experiments have been performed in order to better determine the value of $\beta_{\text {marg }}$ and its dependence on the main plasma parameters.

In previous JET experiments, slow power ramp-down has enabled us to show that the stabilising terms were necessary to explain the time evolution of the saturated island width [11]. In the experiments described here (section 2), we shall show that the marginal beta limit for the $3 / 2$ modes is very low, confirming the large hysteresis observed in earlier experiments, and is near the beta value at the $\mathrm{H}-\mathrm{L}$ transition in typical ELMy H-mode discharges in JET with $q_{95} \approx 3.3$. This means that H-modes are metastable to $3 / 2$ NTMs and their existence depends only on the presence of a large enough seed island. In section 3 detailed modelling of the time evolution of 
the island width is discussed. It shows that the marginal island width is $2-4 \mathrm{~cm}$ and the saturated width below which stabilising terms are important is of the order of 5$6 \mathrm{~cm}$. The relatively large difference between these two values is because the $\chi_{\perp}$ model [13] is more important in this range $(4-6 \mathrm{~cm})$, while the polarisation model can better describe the rapid decay at small island width $w$, when $\beta<\beta_{\text {marg }}$. Constraints on the stabilising terms are examined in section 4 directly from the main feature of the modified Rutherford equation. Finally in section 5 the range of parameters studied in these experiments are discussed.

\section{Power ramp-down experiments}

In the experiments presented here, the NTM is first destabilised, typically with a relatively fast Neutral Beam Injection (NBI) power ramp-up, then the power is kept constant for a couple of seconds for the mode to reach full saturation, finally the power is reduced slowly until $\beta \leq \beta_{\text {marg }}$ and the mode is stabilised.

\subsection{Physics model}

Let us first define a few useful terms and recall the theory of NTM island time evolution as given by the modified Rutherford equation $[1,14,15,16]$, written as an equation for the island growth rate:

$$
\begin{aligned}
\frac{d w}{d t}=\frac{\rho_{s}}{\tau_{\mathrm{R}}}\left[\rho_{s} \Delta^{\prime}(w)+\rho_{s} \beta_{p}(\right. & a_{\mathrm{bs}}\left(\frac{f_{\mathrm{bse}} w}{w^{2}+w_{d, e}^{2}}+\frac{\left(1-f_{\mathrm{bse}}\right) w}{w^{2}+w_{d, i}^{2}}\right) \\
+ & \left.\left.\frac{a_{\mathrm{GGJ}}}{\sqrt{w^{2}+0.2 w_{d, e}^{2}}}+a_{\mathrm{pol}} \frac{w}{w^{4}+w_{d, p o l}^{4}}\right)\right],
\end{aligned}
$$

where $\rho_{s}$ is the radial coordinate of the mode localisation (where $q=m / n$ ), $\tau_{\mathrm{R}}$ the resistive time, $\Delta^{\prime}$ the usual tearing parameter modified mainly by the current gradient, $a_{\mathrm{bs}}, a_{\mathrm{GGJ}}$, and $a_{\mathrm{pol}}$ are parameters described in reference [1]. The first term on the righthand side (rhs) is the usual driving term for the conventional tearing mode and will be assumed here as stabilising. It can be responsible for triggering NTMs [15] but becomes stabilising at large island width due to its $w$ dependence [15]. The second term is the driving term due to the perturbed bootstrap current, which is small at small $w$ due to finite perpendicular transport (the terms $w_{d}$ ) [13]. As a model for the effect of finite ion banana width on the contribution of the ion profiles to the bootstrap current inside the island has been proposed recently [16], we have divided the bootstrap term between the electron $\left(n_{e}, T_{e}\right)$ and ion $\left(n_{i}, T_{i}\right)$ contributions, in relative proportion according to $f_{\mathrm{bse}}$. The value of $w_{d, i}$ is proportional to the ion banana width $\rho_{b}=\sqrt{\epsilon} \rho_{p}: w_{d, i}=\sqrt{(28) \rho_{b}}$ (reference [16] with $w$ as full island width), where $\rho_{p}$ is the poloidal ion Larmor radius and $\epsilon$ is the inverse aspect ratio. As $\rho_{b} \approx 1 \mathrm{~cm}$ in these discharges and in most tokamaks, $w_{d, i} \approx 5 \mathrm{~cm}$ which is relatively large. On the other hand the gradients in the electron profiles contribute to typically $f_{\text {bse }}=70-90 \%$ of the total bootstrap current. The third 
stabilising term is the Glasser-Greene-Johnson term due to curvature effects for which a new dependence at small $w$ has been proposed [14]. The last term is the polarisation term, which has a polarity dependent on mode frequency with respect to the diamagnetic frequencies [17]. This term is assumed negligible or stabilising, in standard discharges, otherwise many more modes would be observed experimentally. The dependence of the polarisation term at small $w$ is not determined yet, as the theory has been developed only for $w>\rho_{b}$. An empirical dependence has been introduced, with the term $w_{d, p o l}$, in order to be able to avoid unphysically large terms at small $w$. The term $w_{d, p o l}$ will be taken as zero or $w_{d, p o l}=\rho_{b}$, as suggested in reference [15]. Note that in the discharges discussed in this paper, $w_{d, p o l} \approx 1 \mathrm{~cm}$, so has typically an effect only for $w \leq 2 \mathrm{~cm}$. For completeness equations (4) of reference [1] are given here as well:

$$
\begin{aligned}
a_{G G J}= & 6 \frac{D_{R}}{\beta_{p}} \approx 6 \frac{\epsilon^{2}}{s} \frac{L_{q}}{-L_{p}}\left(1-\frac{1}{q^{2}}\right), \\
a_{b s}= & a_{2}\left(-L_{b s}\right) \frac{L_{q}}{-L_{p}}, \\
w_{d}= & 5.1 \rho_{s}\left(\frac{q}{\epsilon s m}\right)^{1 / 2}\left(\frac{\chi_{\perp}}{\chi_{\|}}\right)^{1 / 4}, \\
a_{p o l}= & a_{3}\left(\frac{L_{q}}{-L_{p}}\right)^{2} \rho_{p}^{2} g\left(\epsilon, \nu_{i i} *\right) ; \\
& g\left(\epsilon, \nu_{i i} *\right)= \begin{cases}\epsilon^{3 / 2} & \text { if } \nu_{i i} * \leq 0.3 \\
1 & \text { otherwise }\end{cases}
\end{aligned}
$$

where $L_{p}$ and $L_{q}$ are the pressure and q profiles scalelengths, and $L_{b s}$ is the scalelength associated with the contribution of these profiles to the bootstrap current [1].

Typical values of the island growth rates $d w / d t$ versus $w$ are shown in figure 1 for two values of $\beta$ : $\beta_{\text {onset }}$ and $\beta_{\text {marg }}$. Figure 1 is used to define a few terms: $\beta_{\text {onset }}$ is the value of $\beta$ at which the mode onsets, starting from a seed island $w_{\text {seed }}>w_{\text {crit }}$; $\beta_{\text {marg }}$ is the value of beta such that the maximum growth rate is zero and $w_{\text {marg }}$ is the island width at this maximum growth rate. Thus in steady-state, $d w / d t=0$ at $\beta=\beta_{\text {marg }}$ and $w=w_{\text {marg }}$. In the non-steady state case, as will be seen below, the time at which the maximum growth rate is zero does not necessarily correspond to the time at which $w \approx w_{\text {marg }}$. The latter is easily determined experimentally as the growth rate increases or stays constant for decreasing $w$ when $w>w_{\text {marg }}$, but becomes rapidly very negative once $w<w_{\text {marg }}$.

We have avoided the term $\beta_{\text {crit }}$ as it has been used to define both the beta limit associated with beta onset values and the marginal beta limit. The terminology "onset" and "marg" are equivalently defined for $\beta_{N}$ or local and global $\beta_{p}$ values. In figure 1 , the typical time evolution of the growth rate in the discharges discussed below is also sketched (solid line). The mode starts from a given seed island size $w_{\text {seed }}$ at $\beta=\beta_{\text {onset }}$ and evolves up to its saturated state. Then the power is ramped down slowly, so $w$ decreases with $d w / d t \approx 0$ until $w<w_{\text {marg }}$ and $\beta<\beta_{\text {marg }}$ where the mode decays rapidly. The plasma parameters are much better defined at the mode stabilisation as it 
does not depend on the trigger mechanism. Moreover as both $\beta_{\text {marg }}$ and $w_{\text {marg }}$ depend strongly on the stabilising terms in the modified Rurtherford equation, we can obtain information on the importance of these terms. Note that for large $w\left(>>w_{d}\right.$ and $\left.w_{m, p o l}=\sqrt{-3 a_{p o l} / a_{b s}}\right)$, the saturated island width is given by:

$$
w_{s a t \infty}=\rho_{s} \beta_{p} \frac{a_{b s}+a_{G G J}}{-\rho_{s} \Delta^{\prime}}
$$

and thus is proportional to $\beta_{p}$. However when $w$ is of the order of $w_{d}$ or $w_{m, p o l}$, the effective saturated island width is smaller due to the stabilising terms. This break of the co-linearity between $\beta_{p}$ and $w_{\text {sat }}$ can sometimes be observed experimentally and provides an experimental measurement of the island width below which the stabilising terms are contributing significantly, as discussed in the following sections.

In this paper, we are not discussing the conditions at mode onset, however the method used allows us to determine experimentally the conditions near the marginal beta limit that is relevant for the mode onset, apart from the seed island trigger mechanism. Let us clarify somewhat the role of the different effects. The minimum seed island required for a NTM to be destabilised, $w_{\text {crit }}$, depends on the value of $\beta_{\text {onset }}$. On the other hand the size of the seed islands in the discharge depend on $\beta$ and on the physics mechanisms responsible for the island formation. This is why $\beta_{\text {onset }}$ scalings are strongly dependent on $w_{\text {seed }}$ scalings $[2,4]$. However the seed islands do not necessarily continuously increase with increasing $\beta$. The formation of an island perturbing sufficiently the bootstrap current is a complex phenomenon resulting from non-linear interactions between plasma perturbations and the mode itself. Once this interaction and the plasma conditions are such as to form a seed island, say at the $\mathrm{q}=1.5$ surface, its size can be relatively large as compared with the background "noise" level. This seems to be the case for example in the experiments where long sawtooth periods are generated [12]. When the sawtooth period is longer than typically $600 \mathrm{~ms}$, seed islands of $4-5 \mathrm{~cm}$ are generated. However when the sawtooth period is shorter, say $400 \mathrm{~ms}$, no islands are observed, even decaying ones, that is perturbations are below $1-2 \mathrm{~cm}$. This is why, even with a slow $\beta$ ramp-up, $w_{\text {seed }}$ can be much larger than $w_{\text {crit }}$ at

mode onset. To better understand and quantify these effects, it is important to evaluate the different contributions near the marginal beta limit using the method presented in this paper. In this way, when comparing with the onset conditions, one can better measure the role of the trigger mechanism and the size of the seed island. This is somewhat different when the mode is driven unstable first as a conventional tearing mode [15], as the island size is continuously increasing.

\subsection{Typical results and scenarios}

In figure 2 we show two examples of discharges in which a 3/2 NTM has been triggered and later stabilised by slowly decreasing the input power, and thereby the plasma beta. These examples span the range of $B_{0}$ and $I_{p}$ used in these experiments. The ratio $B_{0} / I_{p} \approx 1 T / M A$ is kept approximately constant from $1 \mathrm{~T} / 1 \mathrm{MA}$ to $3.3 \mathrm{~T} / 3.3 \mathrm{MA}$ 
(corresponding to $q_{95} \approx 3.4$ to 3.2 ). Typical parameters at mode onset and stabilisation are given in table 1 for a few discharges in this range. Note that for discharges with $B_{0}<1.7 T$, NTMs can be triggered with NBI only, while for larger toroidal field, long sawtooth period were generated with ion cyclotron heating (ICRH) in order to be able to trigger NTMs at low beta [12]. As shown in figure 2, the mode stabilisation occurs close to the $\mathrm{H}-\mathrm{L}$ transition, at very low marginal beta $\left(\beta_{N, \text { marg }} \approx 0.5-1\right)$. As the discharges are metastable for $\beta>\beta_{\text {marg }}$, this means that in typical JET H-mode discharges, with $q_{95} \approx 3.3$, NTMs are always metastable and their onset only depends on the existence of a large enough seed island and not on the exact value of beta. Of course, as discussed earlier, the size of the seed island might depend on beta and therefore introduce an indirect beta dependence.

In some of the discharges, real-time power control (RTPC) was used to better control the power ramp-down phase, to avoid problems with the vertical feedback system and to prevent mode locking. As soon as the $n=2$ mode is detected above a predefined threshold, the input power waveform is modified such as to reduce $\beta$ quickly, to avoid a large saturated island in the plasma, and then the input power is slowly ramped down in successive steps. This is shown in figure 3 for a discharge with $2.7 \mathrm{~T} / 2.7 \mathrm{MA}$, NBI and central ICRH. The mode is detected at $18.6 \mathrm{~s}$ when the $\mathrm{n}=2$ signal exceeds 0.25 for 200ms, with 6.6MW NBI and 6MW ICRH. Then the real-time control is activated and the ICRH power is reduced to $2.7 \mathrm{MW}$ while the NBI power is kept constant. This is to avoid a large island which would also couple to the wall and reduce its rotation frequency to the range where it strongly interferes with the fast radial field amplifier (FRFA) or could even lock. As the vertical control system of JET does not yet discriminate for $n=2$ perturbations, the resulting excessive switching causes the junction temperature of the FRFA semi-conductors to increase rapidly. The amplifier shuts down causing a disruption if the temperature exceeds $60^{\circ} \mathrm{C}$. As seen in figure 3 , the temperature is kept just below this limit and the end of the discharge is saved for the marginal beta studies. In the last phase, the power is kept constant for three seconds, to let the island settle to a stationary saturated phase, then the NBI power is stepped down by $1.7 \mathrm{MW}, 1.5 \mathrm{~s}$ later the ICRH power is reduced by $1 \mathrm{MW}$ and again $1.5 \mathrm{~s}$ later. Note that due to the link between island width, energy and particle confinement, it does take about $2 \mathrm{~s}$ for the island width and mode frequency to reach a stationary state. The use of the RTPC has allowed a long slow ramp-down phase which will be a key to the detailed studies presented in the next sections. Finally at 25.6s when $\beta_{N}<0.8$, the mode is stabilised (the growth rate becomes very negative). However this does not define the value of $\beta_{N, \text { marg }}$. Indeed, detailed simulations presented below suggest that in fact $\beta_{N}=\beta_{N, \text { marg }}$ at $24 \mathrm{~s}$. Note that similarly to the cases shown in figure 2 , the mode is stabilised just before the H- to L-mode transition which occurs near $26.2 \mathrm{~s}$. It should also be noted that comparable discharges with different $\beta_{N \text {,onset }}$ have similar $\beta_{N, \text { marg }}$, which confirms that the profiles, bootstrap fraction and $\Delta^{\prime}$ are similar for the same $\beta$ and island width. 


\subsection{Collisionality scan and effects at low $q_{95}$}

Only a few discharges have been dedicated to the study of the dependence of $\beta_{N, \text { marg }}$ on density, thus on collisionality and $\rho *$. We show in figure 4 three cases with 2.7T/2.7MA: discharges 53290 (solid), 53284 (dashed) and 53634 (dash-dotted line) in order of decreasing line-averaged densities respectively. Note that the shot 53634 is the one described in figure 3. The waveforms of the total input power are very similar as seen from the similar $\beta_{N}$ time evolution. The time traces of $\rho *$ and $\nu_{i i} *$ are also shown in addition to the line-averaged density. From these parameters one would expect the discharge \#53290 to be the most different in both $\rho *$ and $\nu_{i i} *$, as seen in figure 4. However the time evolution of the island widths shows that they are all very similar. The time slices at $24 \mathrm{~s}, 24.7 \mathrm{~s}$ and $25.5 \mathrm{~s}$ correspond to the time at which $\beta=\beta_{\text {marg }}$ as obtained from the modelling of $w(t)$ discussed in the next section. It shows that $\beta_{N, \text { marg }}$ is between 0.83 and 0.9 for these three discharges, indeed very similar. A relatively large range of $\rho *$ and $\nu_{i i} *$ values has already been obtained as will be discussed in section

5. However further detailed experiments are needed. From these three examples, one obtains that $w_{\text {marg }} \approx 2-4 \mathrm{~cm}$ and the stabilising terms are playing a role up to $5-6 \mathrm{~cm}$. These island widths are actually similar for low and high magnetic field cases, as shown in figure 2. The time evolution of these three cases will be discussed in more detail in the next section.

In figure 5 we show an example of a discharge with a low $q_{95}(2.5)$. It has been shown that the onset beta limit for discharges with $q_{95}<3$ is lower and more disruptive $[18,3]$. This can be due to either a better coupling to sawtooth activity or lower marginal beta limit. We have used the RTPC to decrease rapidly the total input power and avoid mode locking. As can be seen in figure 5, the mode stays until after the H-L transition, while its mode frequency decreases strongly as well. Therefore $\beta_{\text {marg }}$ is low in such scenarios, but the coupling to sawteeth and the creation of a large enough seed island is strong as well. This latter remark is confirmed by the fact that the mode is triggered at the first sawtooth crash with $\beta>\beta_{\text {marg }}$, similar to cases with long sawtooth periods. This suggests that it is the increased coupling to the sawteeth and the large seed island triggered in plasmas with low $q_{95}$ which explain the low $\beta_{\text {onset }}$ observed in previous experiments.

\section{Island width time evolution}

In this section we shall discuss in more detail the time evolution of the saturated island width for the discharges shown in figure 4 and discuss the effects of the stabilising terms on the island evolution near mode stabilisation. We have simulated the time evolution of these three cases using equation (1), first neglecting the polarisation current term $\left(a_{p o l} \approx 0\right)$ and assuming $f_{\mathrm{bse}}=1$ and $w_{d, e}=w_{d, i}=w_{d}$. The results are shown in figure 6 , red curves, and show a very good agreement. 


\subsection{Assumptions for the simulations}

The values of the parameters used in these simulations are given in table 2, where the values in parenthesis are obtained from the experimental profiles. The formula used to obtain these experimental terms are given in reference [1], with the following approximation for the trapped fraction:

$$
f_{t}=1-\frac{(1-\epsilon)^{2}}{(1+1.46 \sqrt{\epsilon}) \sqrt{1-\epsilon^{2}}}
$$

As discussed in reference [19], $f_{t}$ not only depends on $\epsilon$ but also on triangularity. However the above formula has been checked with equilibrium calculations and for standard triangularity it is correct within 10\%, even for a small aspect ratio case, MAST, recently analysed [20]. The term $a_{b s}$ is then given by equation $(2 b)$ with $a_{2}=2.6$ and $L_{b s}=R_{p e}\left(L_{31}+L_{32}\right)$, where $R_{p e}=p_{e} / p$ and $L_{31}, L_{32}$ are obtained from the formulae in reference [19]. The coefficients have been kept fixed in time (except for the dashed line in figure 6(c), as discussed later) and only global $\beta_{p}(t)$ has been used, with the initial boundary conditions given in table 2 . One should actually use the local value of $\beta_{p}$. Similarly one should also include the contributions from density and ion temperature gradients in the bootstrap term as in reference [20]. This would increase $L_{b s}$ by about a factor 1.7. It turns out that the ratio of $\beta_{p}$ to the local poloidal beta, $\beta_{p, l o c}$, is about 1.8 in these discharges, therefore the results and the discussion below would be similar. However the time evolution of $\beta_{p, l o c}$ needs extra smoothing due to the fluctuations in the experimental data. This is why we have preferred to use the global diamagnetic measurement at this stage.

The formula used to calculate $w_{d}$ also needs some clarification. Here we have used the "convective" form, as proposed originally in reference [13] and in a similar way as used in recent Asdex-Upgrade analyses [9]. Introducing a convective form for the parallel transport in the heat equation [13], and defining $w_{d}$ as the island width at which $v_{e} \hat{\mathbf{b}} . \nabla \approx \chi_{\perp} \nabla_{\perp}^{2}$, is in fact similar to assuming the conductive form with:

$$
\chi_{\| c o n v}=v_{T e} \lambda_{\|}=v_{T e} \frac{R_{0} L_{q}}{n w} .
$$

In this way, using the formula in reference [1], we obtain:

$$
w_{d, e}=\left(5.1 \rho_{s}\left(\frac{1}{\epsilon s n}\right)^{1 / 2}\right)^{4 / 3}\left(\frac{\chi_{\perp 93}}{\hat{\chi}_{\| c o n v}}\right)^{1 / 3},
$$

where $\hat{\chi}_{\| c o n v}=v_{T e} R_{0} L_{q} / n$ and the other variables are defined in reference [1]. The values obtained using this definition are of the order of $2-3 \mathrm{~cm}$, whereas it is of the order of $1 \mathrm{~cm}$ using the conductive form [1].

3.2. Effects of the stabilising terms on the time evolution of the saturated width (when $d w / d t \approx 0)$

A usual check to see if a tearing mode has its main drive from the perturbed bootstrap current is to compare the island width time evolution with $\beta_{p}$ as $w_{\text {sat }} \sim \beta_{p}$ at large $w$ 
(equation (3)). This has been done for each case (cyan line, figure 6) and it shows that the island width follows relatively well the scaled $\beta_{p}$ when it has reached saturation. The time at which it is not proportional to $\beta_{p}$ anymore defines the time when the stabilising terms become important. However if one looks in more detail, it is not so straightforward to define this time point and the point when $w=w_{\text {marg }}$. In shots 53290 and 53284, beta drops about at the same time as the island width drops, therefore it is not clear if $\beta_{p}$ becomes smaller than $\beta_{p \text { marg }}$ or if simply the drive decreases. For discharge 53634, on the contrary, the break between the scaled $\beta_{p}$ line (cyan) and $w(t)$, near $23.5 \mathrm{~s}$, is about $2 \mathrm{~s}$ earlier than the rapid stabilisation of $w$. To verify this behavior, we have simulated the island width time evolution without any stabilising terms, setting $w_{d}=0$, and changing $a_{b s}$ to $0.87,0.76$ and 0.91 for figures $6(\mathrm{a})$, (b) and (c) respectively (blue lines). These simulations follow the scaled $\beta_{p}$ as expected, with a delay in the island decay of about $200 \mathrm{~ms}$ due to the factor $\tau_{R} / \rho_{s} \approx 0.2 \mathrm{~s} / \mathrm{cm}$ in the island equation. This confirms the necessity of the stabilising terms to explain the results, as discussed in reference [11], when comparing the simulations shown in red and blue. It should be noted that results similar to the red lines can be obtained assuming $w_{d}=0$ and a finite value of $a_{p o l}$, that is any stabilising term is sufficient. The values of $a_{p o l}$ required in this case are also given in table 2 , where the theoretical value is obtained from equation $(2 d)$, low collisionality, also with $a_{3}=5$.

The above simulations show that the stabilising terms in the modified Rutherford equation explain the change in slope observed in figure $6(\mathrm{a})$ at $t \approx 25 \mathrm{~s}, 6(\mathrm{~b})$ at 25.8 and $6(\mathrm{c})$ at $23.5 \mathrm{~s}$. This happens when the saturated island width is of the order of $2 w_{d}$ and therefore is not anymore just proportional to $\beta_{p}$, but also depends on the stabilising terms. This is typically around $4-6 \mathrm{~cm}$ in JET. One could argue that this change in slope of $w(t)$ is due to modification of the local gradients, which are too difficult to measure. To test this hypothesis, we have simulated the discharge 53634 without any stabilising terms and adding a linear reduction of $a_{b s}$ from $23.5 \mathrm{~s}$ and reaching $40 \%$ at $25.5 \mathrm{~s}$ (dashed blue line). This modification of profiles cannot be resolved from the data without further detailed experiments. As expected it can explain the reduction in island width, but cannot explain the final stabilisation of the mode at 25.8s. Therefore one would still require stabilising terms to explain the final rapid mode decay, albeit at smaller amplitudes as the mode stabilises at a smaller width $(\approx 2.5 \mathrm{~cm})$.

\subsection{Role of ion polarisation and finite island transport terms}

Another question remains which has also motivated this set of experiments: can we differentiate between stabilising terms in the Rutherford equation à la $\chi_{\perp}$ or à la polarisation current models. The aim is to ramp-down the power sufficiently slowly such that the mode stabilises at essentially constant $\beta$, when it is just below $\beta_{\text {marg }}$. Indeed, by measuring $d w / d t(w)$, one could analyse if it is proportional to $w$ or rather to $1 / w^{3}$. The best discharge so far to study this effect is \#53634, which has a long and slow ramp-down phase and in which the mode vanishes while the input power is kept 
constant. Furthermore the mode is stabilised before the H-L transition which occurs just after 26s. However it is very hard to determine $d w / d t(w)$ from the experimental measurement, in particular because the mode stabilises quickly and at low island width, near the measurement noise. The fact that it vanishes quickly would indicate a strong dependence of $(d w / d t)(w)$ on $w$ at small island width, as predicted by the polarisation current model [17]. However the early break of proportionality between $\beta_{p}$ and $w$ noted above, indicates that one should analyse the whole mode evolution more carefully.

First let us analyze the time at which $\beta=\beta_{\text {marg }}$ from the time evolution of the island width shown in figure 6(c) (red line). The best way is to compute when the maximum growth rate crosses zero. This is shown in figure 7 together with the time evolution of the effective island growth rate $d w / d t(w, t)$. First one sees that $\beta=\beta_{\text {marg }}$ at about $24 \mathrm{~s}$, much earlier than the rapid decay. Note that we obtain the same value with the simulation assuming the polarisation current terms as the main stabilising terms. At this time, $w \approx 5 \mathrm{~cm}$ is larger than $w_{\text {marg }}$, since it has not reached steady-state, and therefore the island has a growth rate becoming less negative with a decreasing value of $w$. This explains why $|d w / d t|$ remains small for such a long time. The two curves in figure 7 also determine when $w=w_{\text {marg }}$, i.e. when $d w / d t(w(t))=\max (d w / d t)(t)$, in this case near 25.2s. At this time the growth rate is relatively constant and small, therefore the two curves in figure 7 actually give the time range when $w \approx w_{\text {marg }}$. Then $w$ becomes smaller than $w_{\text {marg }}$, its growth rate becomes more negative with decreasing $w$ and therefore one expects at least an exponential decay of $w$. This gives $w_{\text {marg }} \approx 3.5 \mathrm{~cm}$, but can be in the range $2.5-4 \mathrm{~cm}$ depending on the model used for the stabilising terms. However, as mentioned, the time at which $\beta=\beta_{\text {marg }}$ is not as sensitive and it has been calculated for the three cases shown in figure 6 . These times have been marked in figure 4 .

The comparison of the simulation obtained assuming different stabilising contributions is shown in figure 8 . The value of $\beta_{p}(t)$ used in the simulations is also shown in figure $8(\mathrm{a})$. As mentioned earlier the $w_{d}$ terms (blue line) can better simulate the evolution between 23.5s and 25.5s than the polarisation term (magenta line). However the latter can better model the fast stabilisation observed near 25.8s, figure 8(b), when $w \leq 2.5 \mathrm{~cm}$. This is due to the stronger dependence of $d w / d t$ on $w$ at small $w$ in the polarisation term. The $1 / w^{3}$ dependence also explains why it is more difficult to simulate the effect of stabilising terms between $5-6 \mathrm{~cm}$ and $2.5 \mathrm{~cm}$ with the polarization model only. This general difference between the models will be discussed in the next section, but figure 8 shows that important implications and constraints are obtained on the possible stabilisation mechanisms from these kinds of experiments and simulations. The difference at small island width is further shown in figure 9. Figure 9(a) shows $d w / d t(w, t)$ for the two simulations discussed in figure 8, while figure 9 (b) shows the rhs of the modified Rutherford equation at three time points corresponding to $\beta_{p}=0.42,0.35$ and 0.3. This confirms that independently of the model assumed for the stabilisation terms, $\beta_{p, \text { marg }}$ is about 0.35 in this case, at $24 \mathrm{~s}$. In contrast the value of $w$ when the mode decays rapidly is around $2.5 \mathrm{~cm}$ from the experimental measurement, about $3-3.5 \mathrm{~cm}$ from 
the simulation with the $w_{d}$ terms and about $4 \mathrm{~cm}$ with the polarization term only. This rapid decay is clearly faster when comparing the magenta and the blue lines in figure 8 (b), however the difference in figure 9 , that is in the $d w / d t(w)$ dependence, is not very significant. This is why it is difficult to clearly distinguish which model is appropriate based on the experimental measurement of $w(t)$. Further experiments are needed, in particular such that $w_{\text {marg }}$ would be much larger to allow better measurement or at much higher collisionality to test an increased influence of the polarisation term. As these simulations suggest that both stabilising terms are needed to adequately model the experimental island evolution, we have simulated this case with all the stabilising terms (figure 8, green line). Indeed both the break between 23.5s and 25.2s and the rapid decay between 25.2 and $25.8 \mathrm{~s}$ are very well reproduced. The values used in this simulation are the following: $a_{b s}=1.15, w_{d, e}=1.43 \mathrm{~cm}, a_{p o l}=-1.17 \mathrm{~cm}^{2}, \tau_{R}=10 \mathrm{~s}, a_{G G J}=-0.15, \rho_{s}=$ $58.5 \mathrm{~cm}, \rho_{\mathrm{b}}=1 \mathrm{~cm}, \mathrm{w}_{\mathrm{d}, \mathrm{i}}=\sqrt{28} \rho_{\mathrm{b}}, \mathrm{f}_{\mathrm{bse}}=65 \%$. In this case all the coefficients are close to the expected values, within the error bars which are mainly due to evaluation of the profiles gradients. This further indicates that the polarisation term should be smaller, with the factor $a_{3} \approx 1$ instead of 5 , in order to be important only in the latter phase when $w \leq 3 \mathrm{~cm}$. On the other hand the value of $w_{d, e}$ is consistent with expectations, lying in between the conductive and the convective forms discussed earlier. In the next section we shall discuss the expected range of the stabilising terms which can be inferred from the evolution of the experimental island width and a general discussion of the modified Rutherford equation.

\subsection{Stabilisation of the 3/2 mode by the 4/3 mode}

In figures 6 to 9 we have concentrated on the simulation of the island width evolution near the mode stabilisation. In discharges 53290 and 53634, the modes are actually triggered much earlier. However they are decaying for a while before starting to grow again at the times where the simulation have been started in figure 6 and given in table 2. This is shown in figure 10 where we have simulated the whole $3 / 2$ NTM time evolution (solid blue line), reducing only slightly the $a_{b s}$ (1.05) and $w_{d}(2.5)$ coefficients with respect to the red curve of figure 6(a). There is a clear and discontinuous difference between the simulation of the island width evolution using equation (1) and the experimental island width. The time slices shown by solid lines mark the period when the growth rate is negative. It corresponds to the time between two consecutive sawtooth crashes, as shown by the central SXR trace (green), which also shows that the mode onsets at a sawtooth crash. The spectrogram during this period is shown in figure 10(b). The 3/2 NTM is the main mode and one can see its harmonic. A 4/3 mode is destablised by a first sawtooth crash and stabilised by a second crash. During this time the $3 / 2$ mode amplitude decays strongly. This is similar to the effect of mode decay due to non-resonant perturbed magnetic fields as shown in Asdex-Upgrade [21, 22]. As shown in figure $6(\mathrm{a})$, the mode amplitude follows well the modified Rutherford equation again as soon as the $4 / 3$ mode is stabilised, as the initial condition for the simulation 
in figure 6(a) corresponds to the second solid line in figure 10. Therefore the full island time evolution including the intermediate decay can be explained with equation (1) and an additional stabilising contribution between $21.78 \mathrm{~s}$ and $22.45 \mathrm{~s}$, due to the presence of the $4 / 3$ mode. Detailed modelling of such cases can provide useful information on the strength of the non-resonant coupling. In this case, we have added the drag as an additional term, $\rho_{s} \Delta_{43}^{\prime}$, in the term in square brackets in equation (1). A value of -1.9 has been used in the simulation shown in figure 10(a) (red dashed line), which indeed allows the full time evolution to be simulated. This extra term is actually relatively well constrained, within $10 \%$, and is relatively large as it is of the order of half the main $\Delta^{\prime}$ term. Note that it can be estimated from the linear damping rate just after $21.78 \mathrm{~s}$ and we obtain -1.5. Comparison with theoretical estimates of this additional drag can therefore give an important test of the theory, but is out of the scope of this paper.

\section{Generic constraints on the stabilising terms}

The results presented in the previous section indicate that a very complex time evolution of the $3 / 2$ NTM island width can be modelled using equation (1) with constant parameters, except for $\beta_{p}(t)$. These parameters are in good agreement with expected values, however the formulas used to predict these parameters often have a free constant or an emprical one. For example $\rho_{s} \Delta^{\prime}$ is approximated by $-m$ and its $w$ dependence is often neglected (except in [15]). While $\tau_{R}, f_{b s e}, \rho_{b}$ and $a_{G G J}$ are well defined and can be calculated from the reconstructed equilibrium and plasma profiles, the exact form of $w_{d, e}$ and $a_{p o l}$, as well as the coefficients $a_{2}$ for $a_{b s}$ and $a_{3}$ for $a_{p o l}$ [1] are still under investigations for standard and small aspect ratio geometries. Therefore one could argue that with so many free parameters, the above simulations are not yet a quantitative validation of equation (1). In this section we propose to discuss the characteristics of the modified Rutherford equation so as to reduce the number of effective free parameters and to extract general relations between the saturated island width near $\beta_{\text {marg }}$ and the stabilising terms which can be easily verified experimentally without further assumptions. We shall determine the equation for the saturated island width normalized to the width it would have without stabilizing terms. This ratio is equal to one at large $w$ as the stabilising terms are important only at small $w$, which is why we have termed the island width without stabilising terms $w_{\text {sat } \infty}$. With this equation we are able to give constraints on the range of values of the stabilising terms and of the measured saturated width normalized to $w_{\text {sat } \infty}$ without any assumption on the value of $\rho_{s} \Delta^{\prime}$.

As mentioned above, the least known quantity is the value of $\Delta^{\prime}$. However, at large island width, the saturated width is directly proportional to $1 / \Delta^{\prime}$ and therefore imposes a constraint on the range of $\Delta^{\prime}$ values. This can be best encapsulated by rewriting equation (1) using the definition of $w_{\text {sat } \infty}$ given above (equation (3)). In addition it is convenient to introduce the value of the marginal island width when only the polarisation model is considered, namely $w_{m, p o l}=\sqrt{-3 a_{p o l} / a_{b s}}$ (often called $w_{0}$ in the literature). In 
this way we can write equation (1) as:

$$
\begin{aligned}
\frac{1}{-\rho_{s} \Delta^{\prime}} \frac{\tau_{\mathrm{R}}}{\rho_{s}} \frac{d w}{d t} & \equiv f(w) \\
& =-1+w_{\text {sat } \infty}\left[\frac{f_{b s e} w}{w^{2}+w_{d, e}^{2}}+\frac{\left(1-f_{b s e}\right) w}{w^{2}+w_{d, i}^{2}}-\bar{a}_{G G J} g(w)-\frac{w_{m, p o l}^{2}}{3 w^{3}}\right],
\end{aligned}
$$

where $\bar{a}_{G G J}=-a_{G G J} /\left(a_{b s}+a_{G G J}\right)>0$ and $w_{d, p o l}$ is neglected as we shall consider only the cases with $w \geq w_{\text {marg }}$, so that $w_{d, p o l}^{4}<<w^{4}$. Let us normalize the lengths with $w_{\text {sat } \infty}: \hat{w}=w / w_{\text {sat } \infty}$. The GGJ term can be simplified by noting that $0.2 \hat{w}_{d, e}^{2}<<\hat{w}^{2}$ for $\hat{w}>0.2$ and typical values of $\hat{w}_{d, e}$. Thus $g(w)$ becomes:

$$
g(w)=\frac{f_{b s e} w_{d, e}^{2}}{w\left(w^{2}+w_{d, e}^{2}\right)}+\frac{\left(1-f_{b s e}\right) w_{d, i}^{2}}{w\left(w^{2}+w_{d, i}^{2}\right)} .
$$

Combining the different terms, one obtains the following equation for the saturated island width $\hat{w}=w_{\text {sat }} / w_{\text {sat } \infty}$ (such that $d \hat{w} / d t=0$ ) including the stabilising terms:

$f(\hat{w})=-1+\frac{f_{\text {bse }}\left(\hat{w}^{2}-\bar{a}_{G G J} \hat{w}_{d, e}^{2}\right)}{\hat{w}\left(\hat{w}^{2}+\hat{w}_{d, e}^{2}\right)}+\frac{\left(1-f_{b s e}\right)\left(\hat{w}^{2}-\bar{a}_{G G J} \hat{w}_{d, i}^{2}\right)}{\hat{w}\left(\hat{w}^{2}+\hat{w}_{d, i}^{2}\right)}-\frac{\hat{w}_{m, p o l}^{2}}{3 \hat{w}^{3}}=0$.

\subsection{Range of values allowed for the $w_{d}$ terms}

To analyze in a simple way the above equation, let us evaluate the expected range of the different parameters. By definition $0 \leq \hat{w} \leq 1$, while $\bar{a}_{G G J}$ is typically around 0.1-0.2 for standard aspect ratio tokamaks. The coefficients for electron bootstrap current are larger than for the ions, $f_{b s e}$ is typically between 0.6 to 1 . As $w_{d, e}$ is between $1-3 \mathrm{~cm}$ (in most tokamaks) and $w_{\text {sat } \infty} \approx 4-6 \mathrm{~cm}$ (in the cases discussed here, near $\beta_{\text {marg }}$ ), one expects $\hat{w}_{d, e} \approx 0.2-0.7$. The value of $w_{d, i}$ is around $5 \mathrm{~cm}$, therefore $\hat{w}_{d, i} \approx 1$. Finally the range of $w_{m, p o l}$ is not known but the values used in the above simulations correspond to $w_{m, p o l} \approx 1.5-4 \mathrm{~cm}$, thus $\hat{w}_{m, p o l} \approx 0-1$. figures 11 and 12 show a series of solutions for $w_{d, e}(\hat{w})$ and $w_{m, p o l}(\hat{w})$ to equation 9 for different values of the other parameters.

The first set shows $\hat{w}_{d, e}$ versus $\hat{w}$ for $f_{b s e}=1$, figure $11(\mathrm{a})$, with $\bar{a}_{G G J}=0.1$ (solid lines). Three values of $\hat{w}_{m, p o l}$ have been used: $0,0.4$ and 0.6. The first case, blue solid line, corresponds to the "standard $\chi_{\perp}$ model" assuming $a_{\text {pol }}=0$. This shows that when $\hat{w} \leq 0.5$, i.e. $w_{\text {sat }} \leq w_{\text {sat } \infty} / 2$, the mode is stabilised (as the left most points correspond to the situation just before full stabilisation). Of course, if the polarisation term is added, the mode is stabilised earlier and $\hat{w} \geq 0.6-0.7$. The dashed and dash-dotted lines show that the effect of $\bar{a}_{G G J}=0$ and 0.3 , respectively, is not significant. Figure 11(b) shows the effect of a finite ion bootstrap current contribution, assuming $f_{b s e}=0.7$. Three values of $\hat{w}_{d, i}$ are used, 0 (blue), 1 (red) and 4 (green line) for two values of $\hat{w}_{m, p o l}$ : 0 (solid lines) and 0.4 (dashed lines). A large value of $\hat{w}_{d, i}$ cancels the ion drive therefore the green line starts from $\hat{w} \leq f_{b s e}$. As mentioned above the effect of $\hat{w}_{d, e}$ is such that the mode is stabilised at about half maximum possible width (i.e. without stabilising 
terms), thus $\hat{w}$ can reach $f_{b s e} / 2$ in this case before it is fully stabilised. As expected, with $\hat{w}_{m, p o l}=0.4$, full stabilisation is obtained at smaller values of $\hat{w}_{d, e}$ and the range of saturated island widths is smaller.

Figure 11 shows in addition that a mode has a finite saturated width for $\hat{w}_{d, e} \leq 0.5$. Moreover this saturated width $\hat{w}$ is larger than 0.5 or slightly less if $f_{\text {bse }}<1$, therefore $w \geq w_{d, e}$. It should be emphasized that a good estimate of $w_{s a t \infty}(t)$ is easily obtained experimentally. Indeed it is directly given by the scaled $\beta_{p}$ curves (cyan) shown in figure 6 (if the reference point corresponds to a large enough saturated width). As mentioned earlier these curves do correspond to the island time evolution without any stabilising terms, therefore to $w_{\text {sat } \infty}(t)$. For example for discharge \#53634 at $25 \mathrm{~s}$, figure $6(\mathrm{c}): w_{\text {sat } \infty} \approx 5 \mathrm{~cm}$ and $w=4 \mathrm{~cm}$, yielding $\hat{w}=0.8$. This implies from figure 11(a) that $w_{d, e} \approx 0.4 w_{\text {sat } \infty} \approx 2 \mathrm{~cm}$, if no other stabilising terms are important. Moreover $w_{d, e}$ cannot be larger than $w_{\text {sat } \infty} / 2 \approx 2.5 \mathrm{~cm}$, otherwise the mode would be stable. In addition, if $f_{\text {bse }} \approx 0.7$ as is the case for this discharge, then this gives $w_{d, e} \approx 1.5 \mathrm{~cm}$ for $\hat{w}=0.8$ and $\hat{w}_{m, p o l}=0$. Note that this is close to the conductive form and to the value used in the full simulation (figure 8, green curve). Figure 11(b) also shows the effect of the relatively large value of $\hat{w}_{d, i}(\approx 1$ in this case) found in [16]. It can reduce the island width by $\left(1-f_{b s e}\right)$, so up to $30 \%$ in standard discharges, but then the other stabilising terms have to contribute.

\subsection{Range of values allowed for the $w_{m, p o l}$ term}

The value of $\hat{w}_{m, p o l}$ with respect to $\hat{w}$ is shown in figure 12 . With $f_{b s e}=1$, figure $12(\mathrm{a})$, four values of $\hat{w}_{d, e}$ are taken: 0, 0.2, 0.4 and 0.45. The dashed and dash-dotted lines show the effects of $\bar{a}_{G G J}=0$ and 0.25 , for $\hat{w}_{d, e}=0.4$, which are more important in this case. The blue curve, $\hat{w}_{d, e}=0$, corresponds to the "standard polarisation model". Note that the effect of finite $\chi_{\perp}$ is always present and corresponds, using the minimum estimate for $w_{d, e}$, to $w_{d, e}=0.2$ (red curve). This shows that $\hat{w}_{m, p o l} \leq 0.7$ and the saturated width $\hat{w}$ is larger than 0.7 . Thus in this case also, $w>w_{m, p o l}$. Moreover if an island width $\hat{w} \leq 0.7$ is measured then $\hat{w}_{m, p o l} \leq 0.3$ and the $\chi_{\perp}$ stabilising terms must contribute significantly. For example in figure $6(\mathrm{c})$, just before stabilisation at $25.5 \mathrm{~s}, \hat{w} \approx 2.8 / 4.8=0.58$. Further experimental results of this ratio can confirm that the main reduction of the saturated island width comes from the $w_{d}$ terms. If one takes into account the effect on the ion bootstrap current, assuming $f_{b s e}=0.7$ (figure 12(b)), the curves are slightly shifted to smaller values of $\hat{w}$ as the bootstrap drive is reduced (because $\hat{w}_{d, i}>\hat{w}_{d, e}$ ). Using figure 12 and the value of $\hat{w}=0.58$, we see that $w_{m, p o l} \leq 0.4$. As $a_{b s}+a_{G G J}$ is typically around $0.7-1.3$, this gives a maximum value for $\left|a_{p o l}\right|$ of 1.6, which is smaller than the values obtained using the relation in [1] and than the values needed to simulate cases shown in figure 6 neglecting the $w_{d}$ terms (see table 2 ). This further confirms that the polarisation term should be smaller, as obtained with the coefficient $a_{3}=1$ instead of 5 . 


\subsection{Implications for the JET cases}

Equation (9) and the results shown in figures 11 and 12 demonstrate that strong constraints on the stabilising terms can be obtained directly from the experimental results of $\hat{w}(t)$ close to the marginal $\beta$ limit. These conditions are obtained without any assumption on the value of $\Delta^{\prime}$, except that it does not change significantly during the power ramp-down phase. As the current redistribution time is relatively long and the change in the island width small, this is a realistic assumption. In addition the values of $f_{b s e}, \hat{w}_{d, i}$ and $\bar{a}_{G G J}$ are well defined and can be calculated from the experimental data. Therefore equation (9) can give a strong constraint on $\hat{w}_{d, e}$ and $\hat{w}_{m, p o l}$ in both standard and tight aspect ratio tokamaks. In particular the results obtained in figures 11 and 12 show that the values of $a_{\text {pol }}$ (table 2) used to simulate the discharges shown in figure 6 , with polarization model only, are too large as they are such that $\hat{w}_{m, p o l} \approx 0.7-0.85$. Therefore, when the polarisation model is considered, finite $w_{d}$ values have to be used, reducing $\hat{w}_{m, p o l}$ by about a factor of 2 , thus $a_{p o l}$ by a factor of 4 . This is consistent with the simulations which suggest that the coefficient $a_{3}$ should be reduced from 5 to 1 as discussed earlier. Figure 11 also shows that the range of $\hat{w}_{d, e}$ is $0.2-0.5$, thus typically $w_{d, e} \approx 0.6-2.2 \mathrm{~cm}$ for the discharges presented here, which is consistent with the range obtained from the values of $w_{d 1}$ and $w_{d 2}$ (table 1$)$.

\section{Preliminary dimensionless parameter scaling of the marginal beta limit}

The values of the main plasma parameters related to NTMs are given in table 1 for discharges spanning this range in field and currents. In figure 13 we show the value of $\beta_{N}$ as a function of $\rho *$ and $\nu_{i i} *$ near the mode stabilisation, that is near the top of the exponential island width decay. This corresponds to the time at which $w \approx w_{\text {marg }}$, but $\beta_{N}\left(w_{\text {marg }}\right)$ can be at values of $\beta$ slightly lower than $\beta_{N, \text { marg }}$ as discussed in the previous section. Figure 13(a) indicates a slightly stronger dependence of $\beta_{N}\left(w_{\text {marg }}\right)$ on $\rho *, \beta_{N}\left(w_{\text {marg }}\right) \sim \rho *_{-3}^{1.1}$ with $\rho *=\rho *_{-3} 10^{-3}$, than from previous fits of $\beta_{N, \text { onset }}$ [11]. As shown by the detailed simulations of the island width time evolution and as mentioned by other authors, $[9,16]$, this is compatible with the $\chi_{\perp}$ model as well as with the polarisation model. Figure 13(b) shows no dependence on $\nu_{i i} *$ once the main $\rho *$ dependence is removed, however further experiments at much larger values of $\nu_{i i} *$ are needed. Indeed this would verify if the polarisation term has any significant influence on marginal beta limit. It should be noted that for most of the discharges shown in figure 13, the mode is stabilised after the $\mathrm{H}-\mathrm{L}$ transition. Therefore it requires a better time resolution of the equilibrium and plasma profiles, in order to accurately evaluate the local parameters. This is why we show here the scaling in terms of global parameters only. This preliminary scaling indicates the value of the seed island control, demonstrated in JET [12], to ITER-FEAT, since it suggests ITER-FEAT H-mode scenarios, with $q_{95} \approx 3$, should be metastable to NTMs. 


\section{Discussion}

We have shown that the marginal beta limit in typical JET H-modes, with $q_{95} \approx 3.3$, is very low and near the H-L transition. Therefore H-modes are metastable to the $3 / 2$ NTMs in JET and preliminary dimensionless scalings indicate this should also be the case in ITER-FEAT, so that the NTM beta-limit is determined by the physics of the seeding process. In many cases the NTM in JET is stabilised after the H-L transition during a faster decrease of $\beta$, therefore leading to larger scatter of the data. With a very slow ramp-down of the input power and therefore of $\beta_{p}$, the mode is stabilised at almost constant power input while still in $\mathrm{H}$-mode, and one can get more insights to the contributions of the different stabilising terms. This indicates that these terms affect the saturated width when $w \sim 4-6 \mathrm{~cm}$ and are such that $w_{\operatorname{marg}} \approx 2-4 \mathrm{~cm}$ for very different cases from $B_{0}=1 T / I_{p}=1 M A$ to $B_{0}=3.3 T / I_{p}=3.3 M A$.

It has also been shown that very slow ramp-down experiments can indicate the influence of the stabilising terms on $w_{\text {sat }}$, for $w_{\text {sat }} \leq 4-6 \mathrm{~cm}$, and can give accurate measurement of the marginal island width, as there is a change of decay rate from constant to exponential. At any given time and $\beta$ value, a small ratio between $w$ and $w_{\text {sat } \infty}(0.5$ or smaller $)$ indicates that the stabilising terms have a weak $w$ dependence and vice-versa when $w / w_{\text {sat } \infty} \approx 1$. This ratio is of course minimum at $w=w_{\text {marg }}$, just before the mode is stabilized. In particular one would expect this minimum ratio to be closer to one when collisionality is increased and this will be the subject of further experimental studies. The comparison of mode stabilisation in standard H-mode discharges with discharges with peaked density which have a stronger bootstrap current due to the contribution of the density gradient might help in separating the effects of collisionality on the driving term with the effects on the stabilising terms.

The aim of these experiments is also to determine the relative importance of the $\chi_{\perp}$ and polarisation model near the marginal beta limit where they play a key role. Detailed simulations of the island evolution during the power ramp-down phase and when the mode is stabilised indicate that the $w_{d}$ terms are the main stabilising contribution in the range $w \geq 3 \mathrm{~cm}$. The effect of a larger effective perpendicular transport for the ions, due the finite banana width, has been considered. It is seen that it can explain a reduction of the bootstrap drive of up to $30 \%$. It cannot explain full stabilisation, but leads to lower values required for the $w_{d, e}$ term. On the other hand, the reduced perpendicular transport effect on the GGJ term leads to an effective $1 / w$ dependence at small to intermediate $\left(<w_{\text {marg }}\right)$ values. This has no strong effects on $w_{\text {marg }}$ or $\beta_{\text {marg }}$, but leads to a faster island decay for $w<w_{\text {marg }}$ even without the polarisation term. However the island decay seems faster and would require an additional stabilisation from the polarisation model when $w \leq 3 \mathrm{~cm}$. Since the fast decay is difficult to measure accurately, this analysis should be performed in scenarios with larger $w_{\text {marg }}$, if possible, and mainly at very high collisionality. Note that this is in contrast with detailed studies performed in Compass-D, where the polarisation term was shown to be the main stabilising contribution. In particular it could explain the $\beta_{p}$ 
and collisionality dependence of the trigger of non-rotating MHD modes with increased error field perturbations [2]. However the coefficient $a_{3}$ of the polarisation term used to explain the observed results would be much too high for JET cases, even though this could be due to the bootstrap form used. The discharges analysed in this paper and the study of the characteristics of the modified Rutherford equation suggest that the relation used to evaluate the polarisation term (equation $(2 d)$ ) should be reduced by a factor of about 5 , setting $a_{3}=1$. The fact that the polarisation term is more important for non-rotating induced seed islands could be due to the mode frequency dependence of the polarisation term. Similar experiments on error field induced modes at high $\beta$ are planned on JET, and possibly other tokamaks, and should provide more information. In particular when combined with a slow ramp-down phase once the mode has saturated to determine the terms near the marginal beta limit.

We have also shown that $\beta_{\text {marg }}$ is similar or slightly lower in discharges with low $q_{95}(\approx 2.5)$. This suggests that the lower $\beta_{\text {onset }}$ observed in these cases is due to the stronger effect of sawteeth in triggering large enough seed islands, which allows onset closer to $\beta_{\text {marg }}$. In particular the mode onsets at the first sawtooth crash when $\beta>\beta_{\text {marg }}$, similar to cases with stabilised sawteeth [12] and to tight aspect ratio tokamaks [20].

Important information and constraints are obtained from the experimental measurement of $\hat{w}(t)=w_{\text {sat }}(t) / w_{\text {sat } \infty}(t)$, which is well approximated using the scaled $\beta_{p}(t)$ curves shown in figure 6 for example. In particular the mode is predicted to be stabilised when $\hat{w}$ is reduced to 0.5 or to $\left(1-f_{\text {bse }}\right) / 2$ when a significant ion bootstrap current contribution is present. The results shown in figures 11 and 12 give the range of values of the stabilising terms as a function of $\hat{w}(t)$ and can be used to test further the validity of the modified Rutherford equation and of the formulae used to evaluate these stabilising terms, both in standard and small aspect ratio tokamaks. In particular, those constraints obtained from the experimental measurement of $\hat{w}(t)$ do not require any knowledge of the value of $\Delta^{\prime}$, a contribution difficult to evaluate from experimental measurements. It is also shown that the polarisation model should not be considered in isolation, as even small values for $w_{d}$ increase the constraints on $w_{m, p o l}$ and $\hat{w}$ at mode stabilisation. In addition these analyses show that the range of values for $w_{d, e}$ given by equation $(2 c)$ and equation (6) is consistent with the values required to influence $w_{\text {sat } \infty}$ when it is of the order of $4-6 \mathrm{~cm}$ and to stabilise the mode when $w_{\text {marg }} \approx 2-4 \mathrm{~cm}$.

In conclusion these experiments and analyses present an important method to validate the NTM theory. Further experiments are planned on JET and other tokamaks to fully exploit this method and should allow better extrapolation to future devices.

\section{Acknowledgments}

We are grateful to the UKAEA for the efficient operation of the JET tokamak. This work was performed under the European Fusion Development Agreement and was supported in part by the Swiss National Science Foundation, in part by EURATOM and in part by the UK Department of Trade and Industry. 


\section{References}

[1] Sauter O. et al, Phys. Plasmas 4 , 1654 (1997).

[2] Gates D. A. et al, Nucl. Fusion 37, 1593 (1997).

[3] Buttery R. J. et al, Plasma Phys. Contr. Fusion 42, B61 (2000).

[4] La Haye R. J. and Sauter O., Nucl. Fusion 38, 987 (1998).

[5] Guenter S. et al, Nucl. Fusion 38, 1431 (1998).

[6] Isayama A. et al, Plasma Phys. Contr. Fusion 42, L37 (2000).

[7] Aymar R. et al, Nucl. Fusion 41, 1301 (2001).

[8] La Haye R. J. et al, Phys. Plasmas 7, 3349 (2000).

[9] Zohm H. et al, Phys. Plasmas 8, 2009 (2001).

[10] Wilson H. R. et al, Phys. Plasmas 3, 248 (1996).

[11] Buttery R. J. et al, submitted to Nucl. Fusion.

[12] Sauter O. et al, Phys. Rev. Lett. 88, 105001 (2002); Westerhof E. et al, submitted to Nucl. Fusion.

[13] Fitzpatrick R. et al, Phys. Plasmas 2, 825 (1995).

[14] Lutjens H. et al, Phys. Plasmas 8, 4267 (2001).

[15] Reimerdes H. et al, Phys. Rev. Lett. 88, 105004 (2002).

[16] Poli E. et al, Phys. Rev. Lett. 88, 075001 (2002).

[17] Connor J. W. et al, Phys. Plasmas 8, 2835 (2001).

[18] Zohm H. et al, Plasma Phys. Contr. Fusion 37, A313 (1995).

[19] Sauter O. et al, Phys. Plasmas 6, 2834 (1999).

[20] Buttery R. J. et al, Phys. Rev. Lett. 88, 125005 (2002).

[21] Yu Q., Gunter S., Lackner K., Phys. Rev. Lett. 85, 2949 (2000).

[22] Gunter S. et al, Phys. Rev. Lett. 87, 275001 (2001). 
Table 1. Typical plasma parameters related to H-L transition and NTM physics at mode onset (on) and stabilisation (off) for discharges spanning the experimental range in field and current, leading to the results shown in figure 13. All are with standard shape and $q_{95} \approx 3.3$, except $\# 53640$ which has $q_{95}=2.5$. The conductive form $w_{d 1}$ $[\mathrm{cm}]$ is given by equation $(2 c)$ and the convective form $w_{d 2}$ by equation (6). Ps [MW] gives $\left(P_{N B I} ; P_{R F}\right)$ at the mode onset and stabilisation. $a_{p o l}\left[\mathrm{~cm}^{2}\right]$ is given by equation $(2 d)$ with $a_{3}=5$ and $\rho *=10^{-3} \rho_{-3}^{*}$. The effective charge number has been included in the evaluation of $\nu_{i i} *$ and $\nu_{e} *$.

\begin{tabular}{lllllllllll}
\hline shot & $I_{p, M A} / B_{0, T}$ & $\beta_{N, \text { on }}$ & $\beta_{N} / \beta_{p, o f f}$ & $\beta_{N, H-L}$ & $P s_{\text {on }} / P s_{\text {off }}$ & $w_{d 1} / w_{d 2}$ & $a_{p o l}$ & $\rho_{-3}^{*}$ & $\nu_{i i} *$ & $\nu_{e} *$ \\
\hline 50683 & $1.1 / 1.1$ & 3.4 & $1.04 / 0.4$ & 0.9 & $(10 ; 0) /(1.4 ; 0)$ & $2 / 4$ & -25 & 5.6 & 0.22 & 0.21 \\
47276 & $1.6 / 1.65$ & 2.59 & $0.98 / 0.36$ & 0.7 & $(12.6 ; 0) /(0 ; 0)$ & $1 / 2$ & -4 & 4.8 & 0.25 & 0.23 \\
53640 & $2.2 / 1.7$ & 1.15 & $0.8 / 0.24$ & 1.17 & $(6.8 ; 0) /(0 ; 0)$ & $1 / 1.8$ & -3.5 & 4.2 & 0.15 & 0.12 \\
53634 & $2.7 / 2.7$ & 1.43 & $0.82 / 0.31$ & 0.59 & $(6.6 ; 6) /(4.9 ; 1)$ & $0.6 / 2$ & -8 & 4.0 & 0.04 & 0.02 \\
53286 & $3.3 / 3.3$ & 1.12 & $0.44 / 0.18$ & 0.59 & $(6.3 ; 6) /(0 ; 0)$ & $0.5 / 1.5$ & -4 & 2.5 & 0.34 & 0.14 \\
\hline
\end{tabular}

Table 2. Parameters of equation (1) used in the simulations presented in figures 6-8, neglecting either the polarization term or $w_{d}$. In parenthesis the values obtained from experimental data.

\begin{tabular}{llllllll}
\hline shot & $a_{b s}$ & $w_{d}[\mathrm{~cm}]$ & $\rho_{s}[\mathrm{~cm}]$ & $\tau_{R} / \rho_{s}[\mathrm{~s} / \mathrm{cm}]$ & $t_{\text {start }}[\mathrm{s}]$ & $w_{\text {start }}[\mathrm{cm}]$ & $a_{\text {pol }}\left[\mathrm{cm}^{2}\right]$ \\
\hline 53290 & $1.19(1.06)$ & $3.1(2.12)$ & 58.5 & $0.20(0.3-0.2)$ & 22.45 & 2.5 & $-4.3(-4.54)$ \\
53284 & $1.03(1.02)$ & $2.4(2.04)$ & 58.5 & $0.15(0.3-0.15)$ & 23.76 & 3.0 & $-3.0(-6.23)$ \\
53634 & $1.12(1.19)$ & $3.0(2.09)$ & 58.5 & $0.20(0.3-0.15)$ & 20.60 & 4.8 & $-5.1(-6.24)$ \\
\hline
\end{tabular}

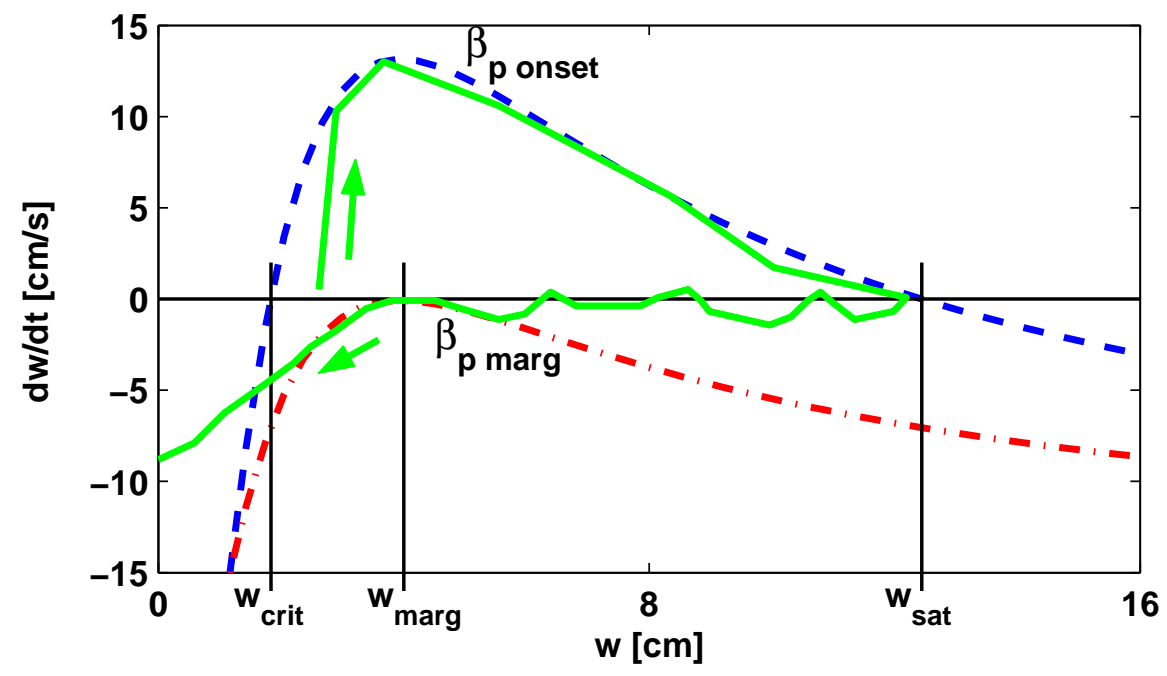

Figure 1. Examples of the $w$ dependence of the rhs of equation (1) for parameters similar to the one obtained in the discharges presented in this paper and for two values of $\beta_{p}, \beta_{p \text {,onset }}$ (dashed line) and $\beta_{p, \text { marg }}$ (dash-dotted). These also defines $w_{\text {crit }}$, which depends on $\beta_{\text {p,onset }}, w_{\text {marg }}$ and $w_{\text {sat }}$. The solid line sketches the typical time evolution of a NTM, while these curves move with the time evolution of $\beta_{p}$. 

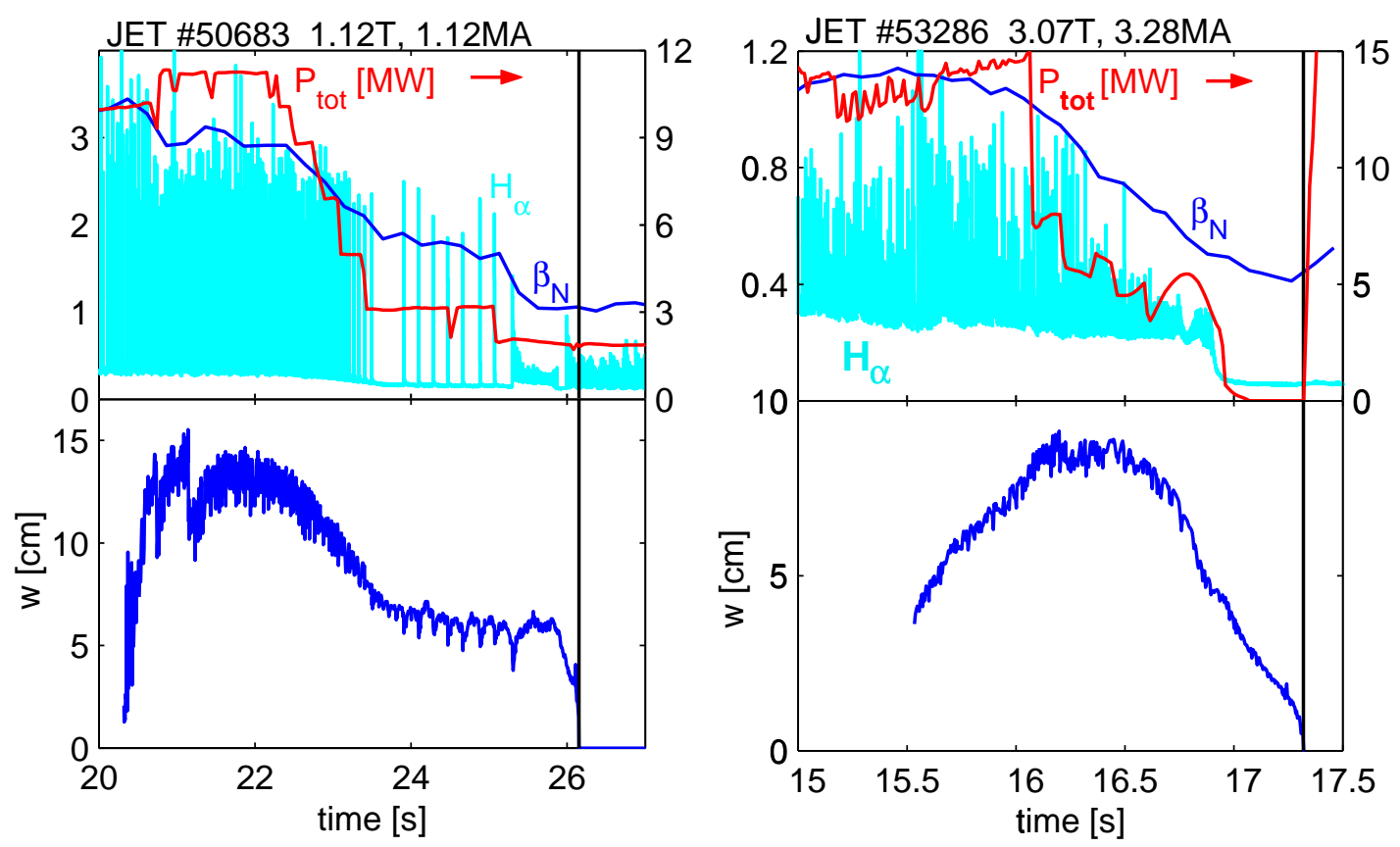

Figure 2. Two examples of mode stabilisation at low and high magnetic field and current, at $q_{95} \approx 3.3$. In all cases, the $3 / 2$ NTM is stabilised just before or after the H-L transition as indicated by the $H_{\alpha}$ trace. 

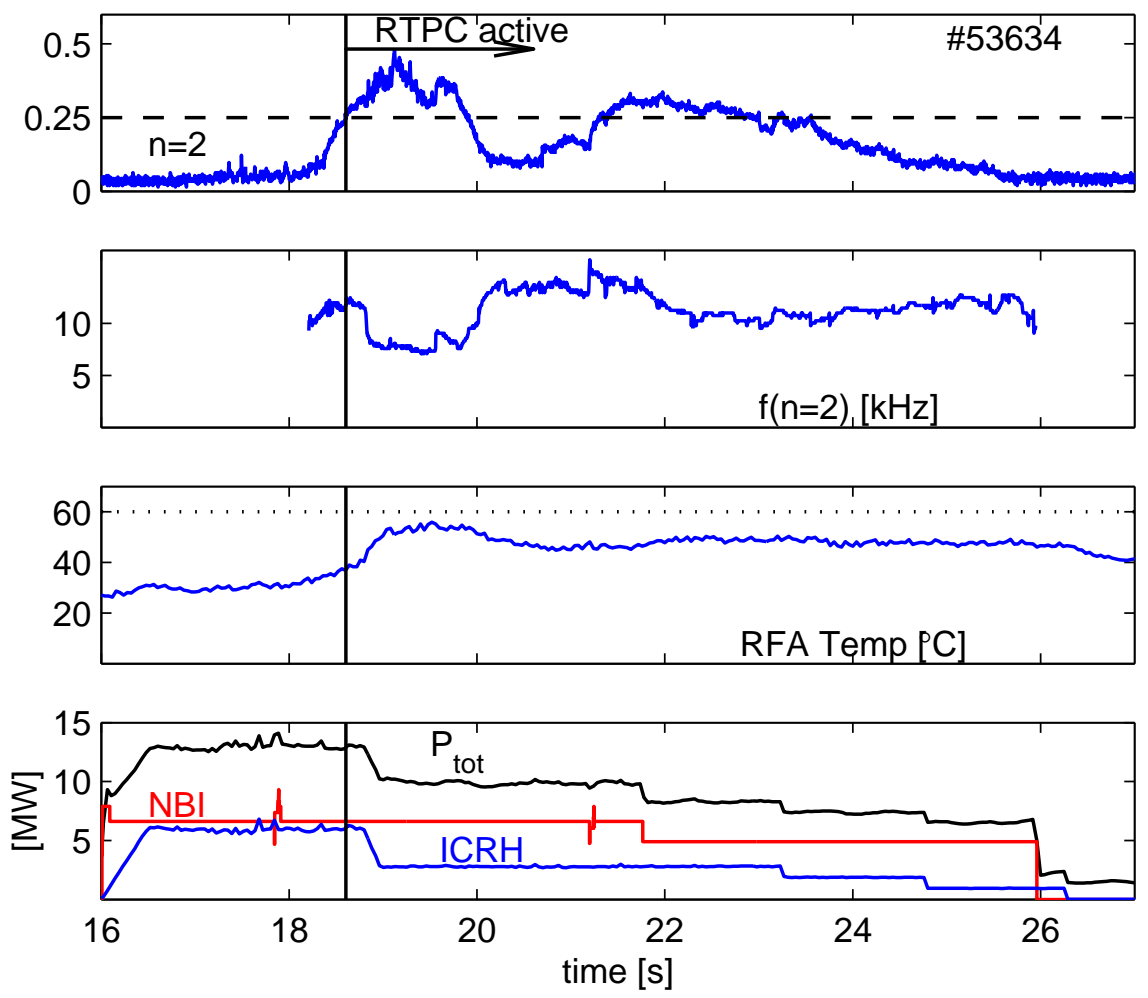

Figure 3. Time traces of the mode onset and slow ramp-down phase of a discharge with 2.7T/2.7MA. The real-time power control system (RTPC) has been used to control a long ramp-down phase. The temperature in the FRFA, $n=2$ magnetic signal, mode frequency and input powers are also shown. After the mode is detected at an amplitude larger than 0.25 , the RTPC system decreases rapidly the ICRH power, then a slow ramp-down phase is controlled with alternate NBI and ICRH step-down. 


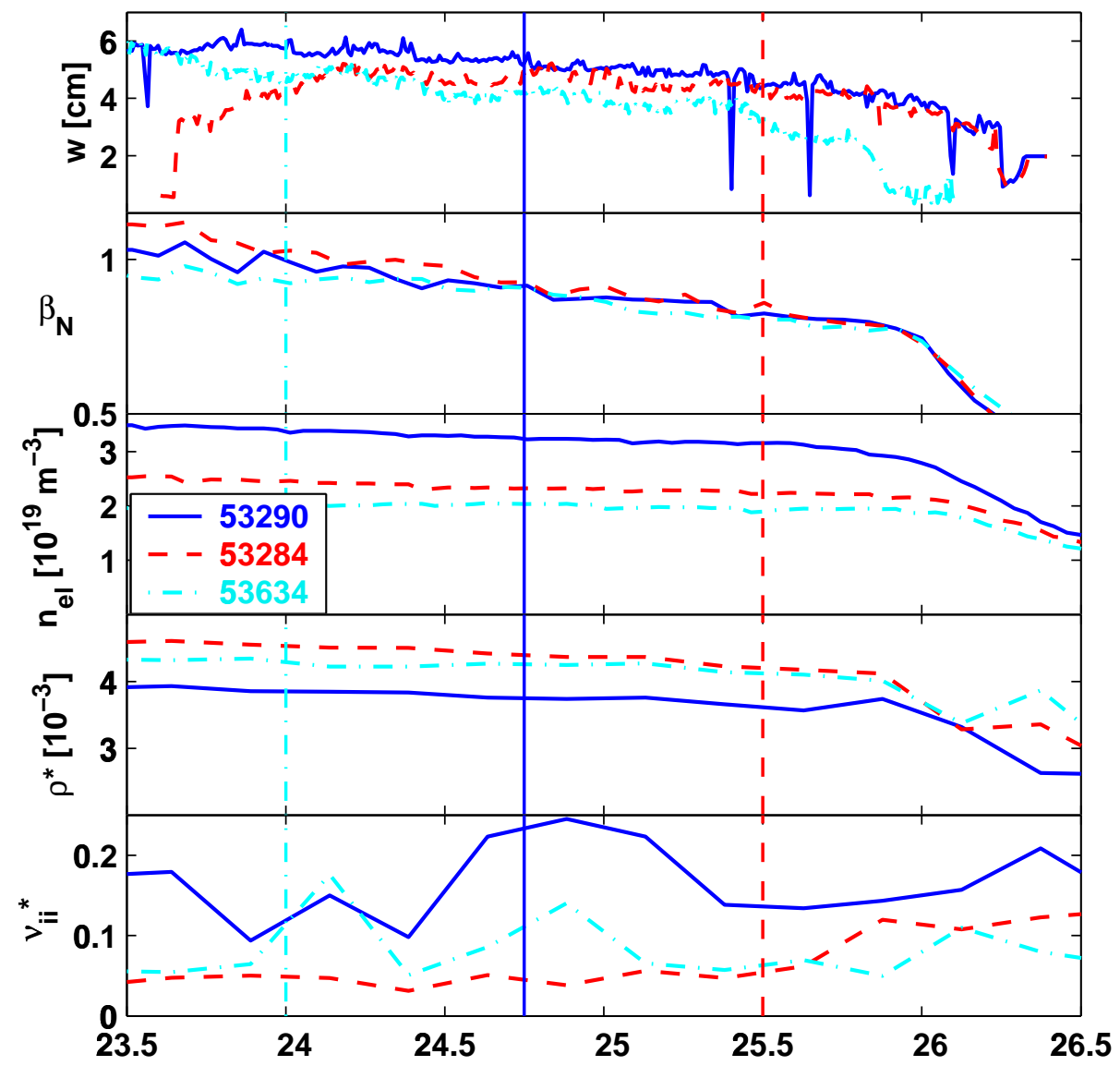

Figure 4. Mode stabilisation for similar discharges, with 2.7T/2.7MA, but with three different line-averaged densities. The island width near mode stabilisation, $\beta_{N}, \rho *$ and $\nu_{i i} *$ are also shown. The time-slices marked by vertical lines correspond to $\beta=\beta_{\text {marg }}$, the time at which the maximum growth rate is zero as given by the rhs of equation (1) and obtained from the simulation shown in figure 7 for \#53634. 


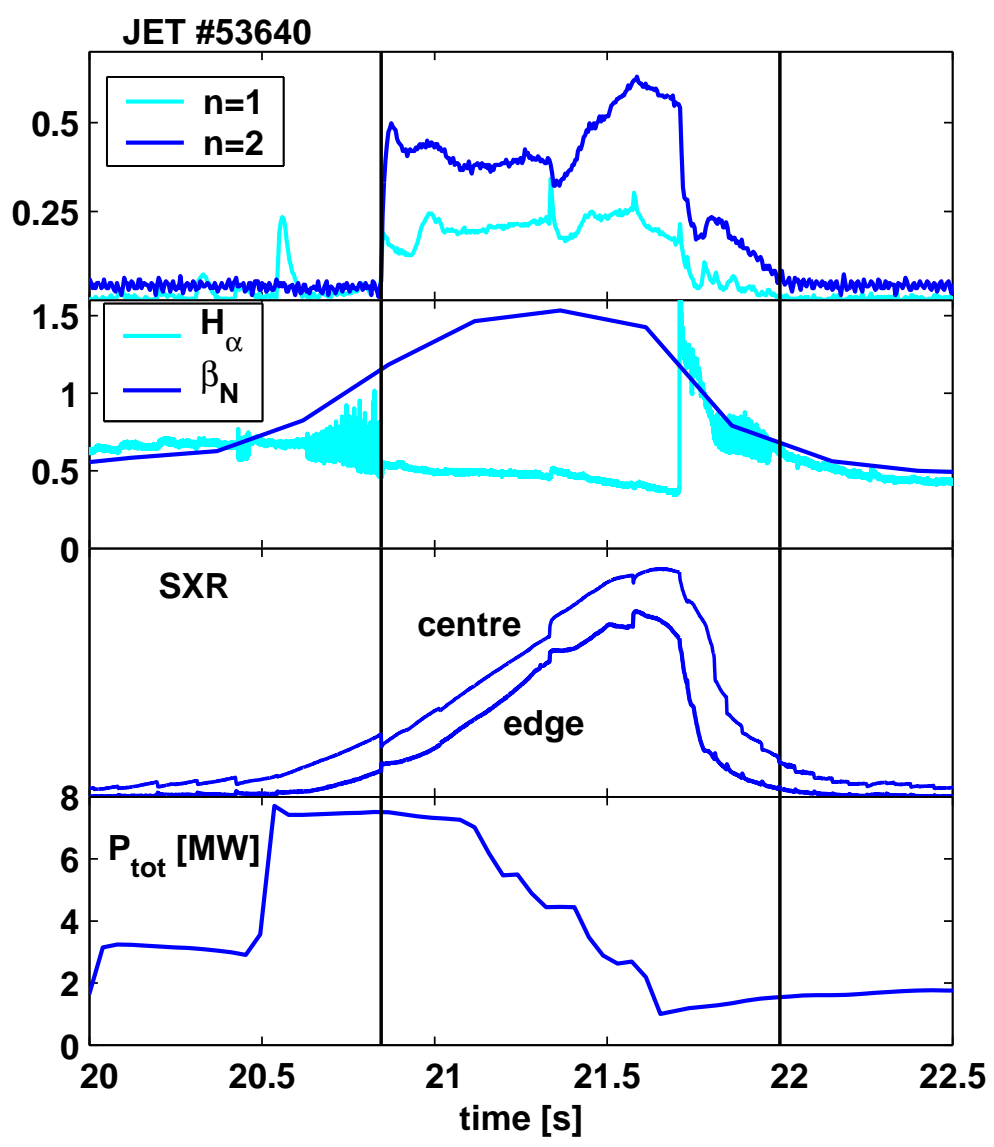

Figure 5. Low $q_{95}, 2.54$, discharge. Mode onsets at a sawtooth crash which might have triggered the $\mathrm{H}-\mathrm{L}$ transition as well. The power is ramped-down quickly after mode onset, using the RTPC feedback, which prevents mode locking. The mode is stabilised at $\beta_{N} \approx 0.65$ well after the H-L transition at $\beta_{N} \approx 1.1$.
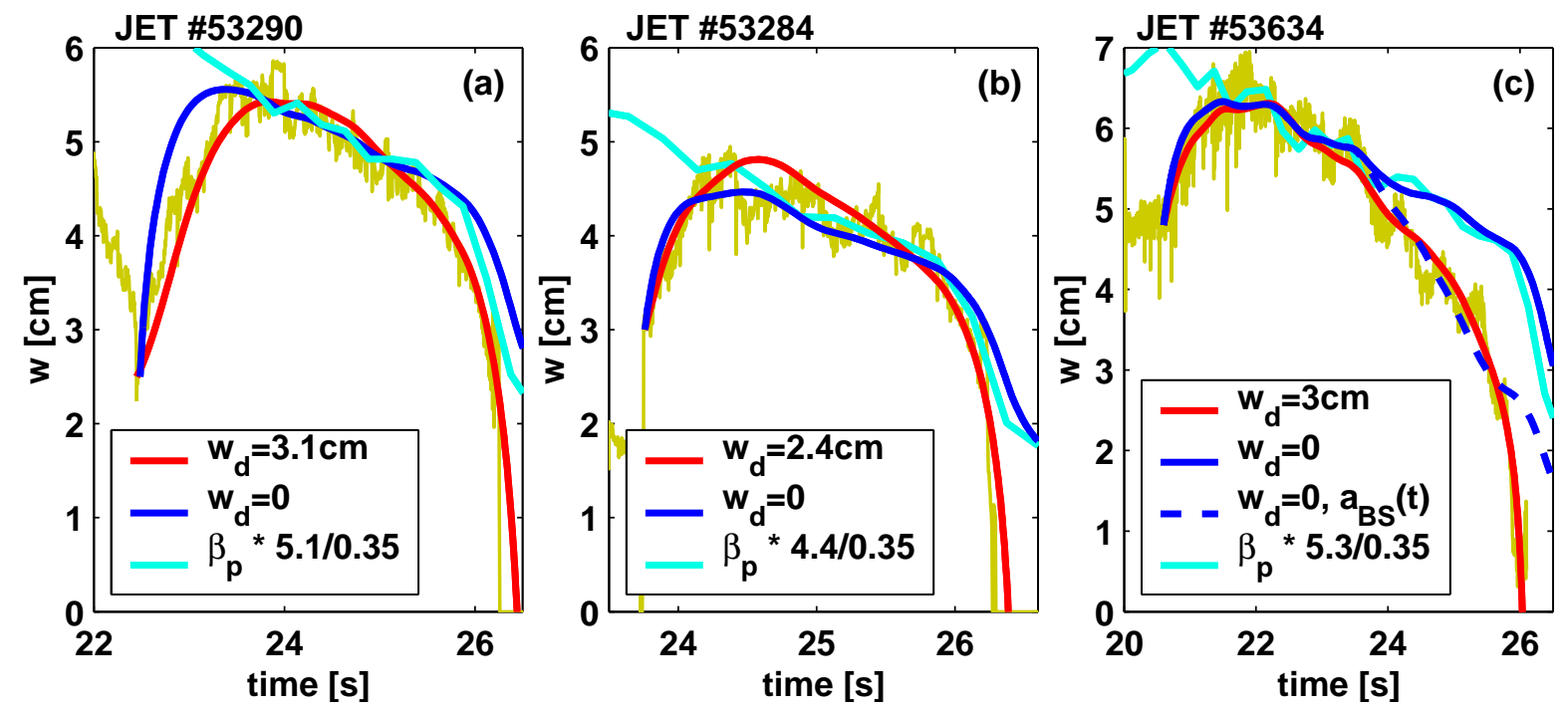

Figure 6. Island width time evolution for the three cases shown in figure 4. The simulation with finite $w_{d}$ (red lines) and no stabilising terms (blue line) are shown as well as a scaled $\beta_{p}$ trace (cyan). The dashed blue line in (c) corresponds to the blue simulation but with $a_{b s}$ decreasing gradually by up to $40 \%$ between $23.5 \mathrm{~s}$ and $25.5 \mathrm{~s}$. 


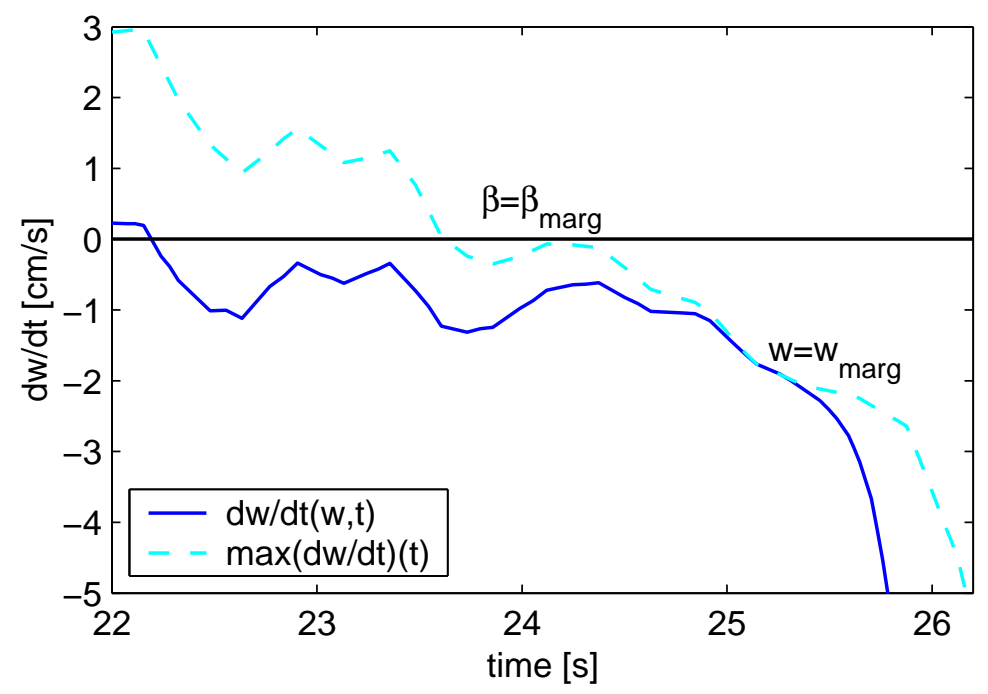

Figure 7. Time evolution of the actual island growth rate $(d w / d t(w, t)$, solid line), corresponding to the red curve in figure 6(c), and of the maximum growth rate, $\max (\mathrm{dw} / \mathrm{dt})(\mathrm{t})$, determined by the maximum of the rhs of equation $(1)$ at each timestep (dashed line). During the time evolution, $\beta \approx \beta_{\text {marg }}$ when the maximum growth rate is zero, while $w \approx w_{\text {marg }}$ when the two curves overlay. These occur at slightly different times because the island is not in steady-state.

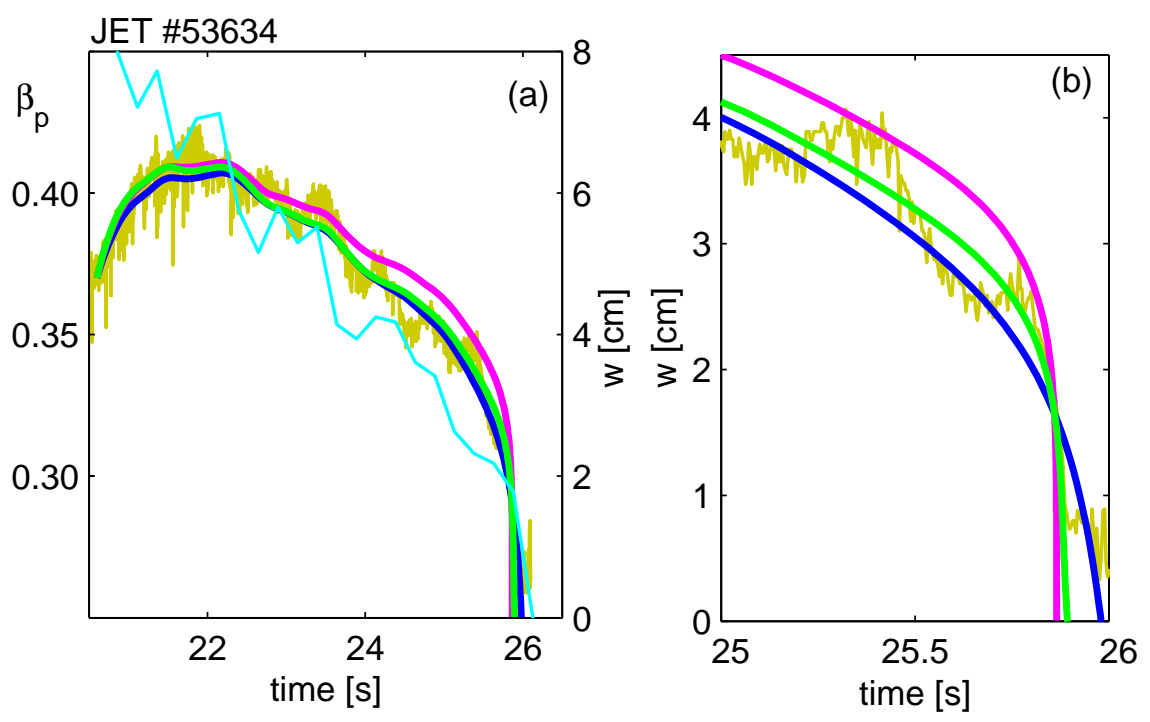

Figure 8. Simulations of the island width evolution with different assumptions for the stabilising mechanisms. $\chi_{\perp}$ model only (blue, $f_{b s e}=1$ ), polarisation model only (magenta), all stabilising terms (green, $f_{b s e}=0.65$ ). (a) Full time evolution and $\beta_{p}(t)$ (cyan). (b) Zoom near mode stabilisation. 

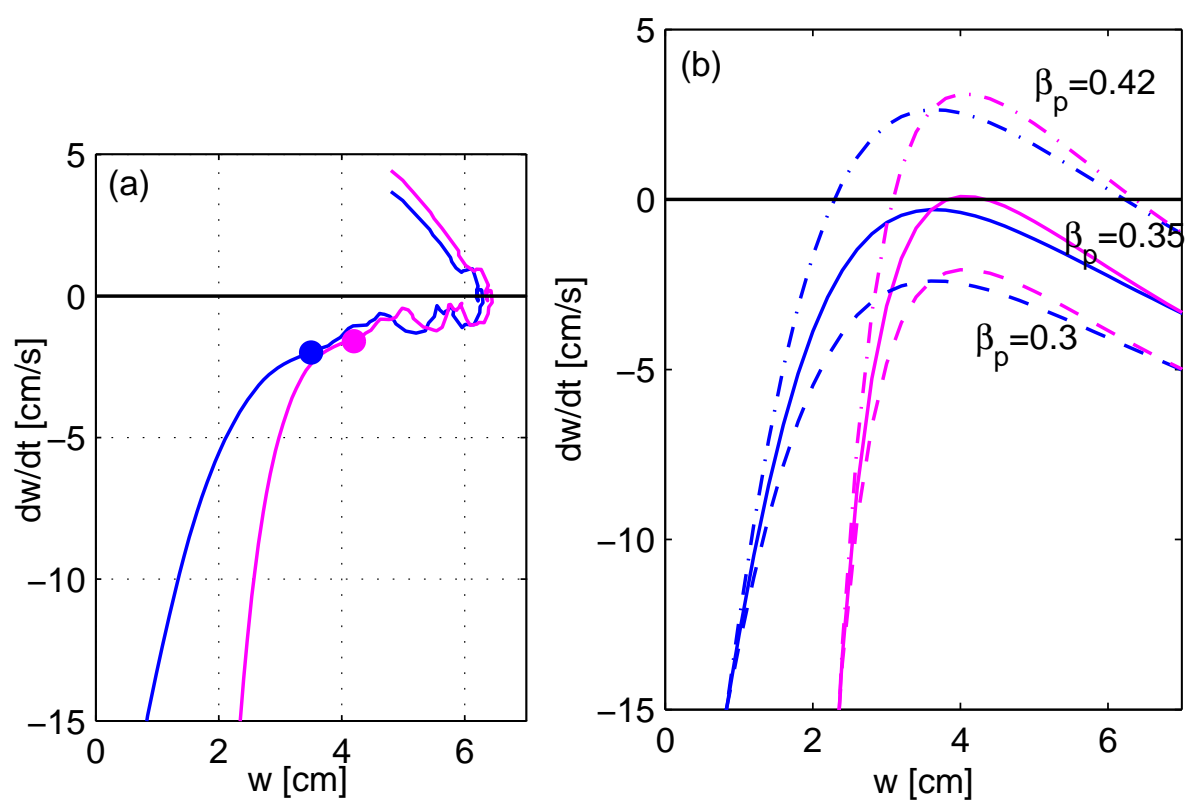

Figure 9. (a) End of island width time evolution for JET \#53634, with the simulation shown in figure 8. The simulation with the $\chi_{\perp}$ model (figure 8, blue) and with the polarisation model (figure 8, magenta) are shown. (b) The rhs of equation (1) with the parameters used in (a) and figure 8, for three different values of $\beta_{p}$ corresponding to $\mathrm{t}=22 \mathrm{~s}, 24 \mathrm{~s}$, and $25.8 \mathrm{~s}$. 


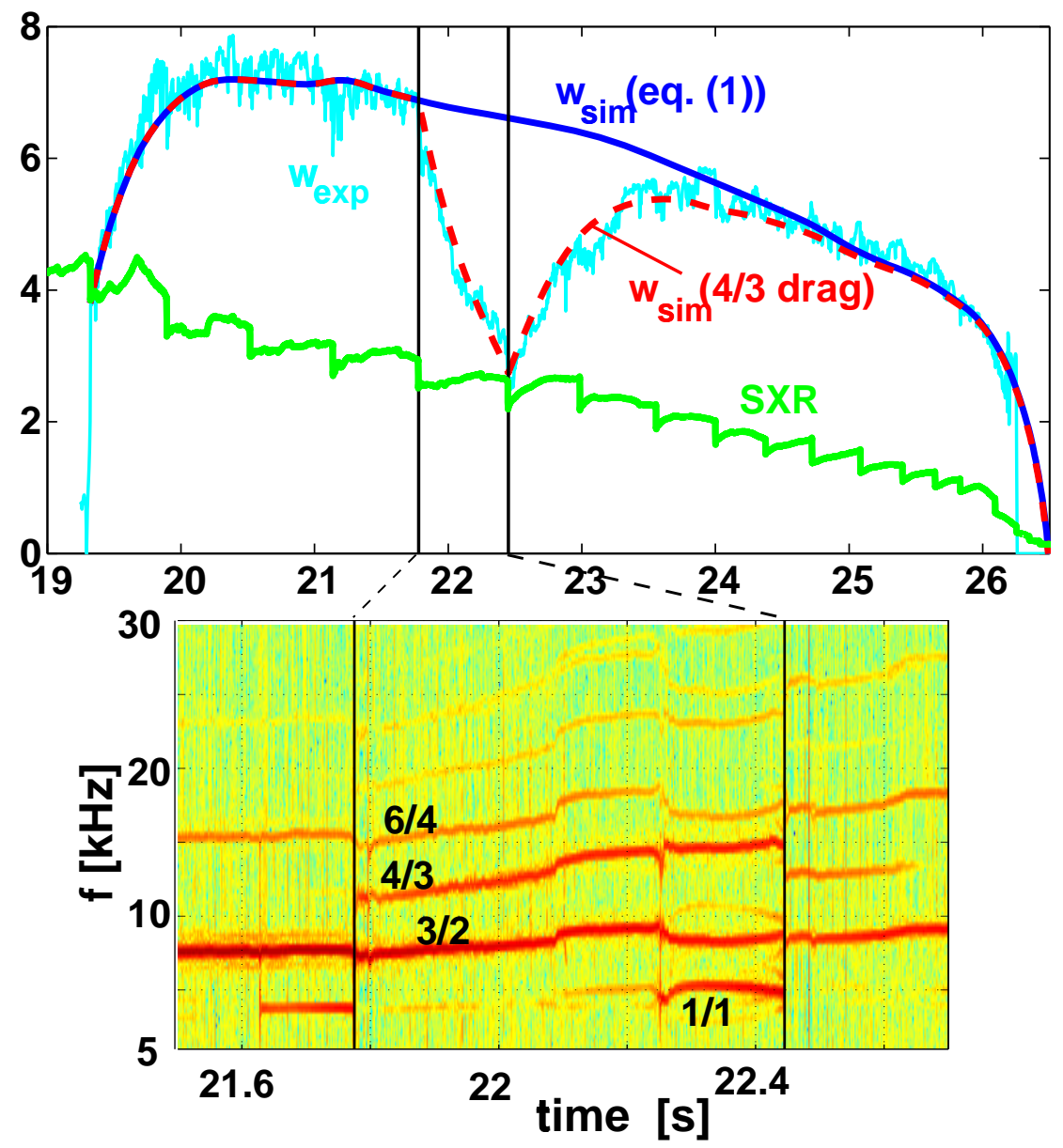

Figure 10. (a) Full island width evolution for JET \#53290, figure 6(a), which clearly deviates suddenly from the simulation using equation (1) between $21.775 \mathrm{~s}$ and $22.445 \mathrm{~s}$. This correlates well with the appearance of a $4 / 3$ mode at the sawtooth crash at $21.78 \mathrm{~s}$ and its disappearance at the next sawtooth crash at 22.45s. Adding an additional drag $\rho_{s} \Delta_{43}^{\prime}=-1.9$ in between the two sawtooth crashes and leaving all the other coefficients constant, reproduces well the full island evolution (red dashed). 


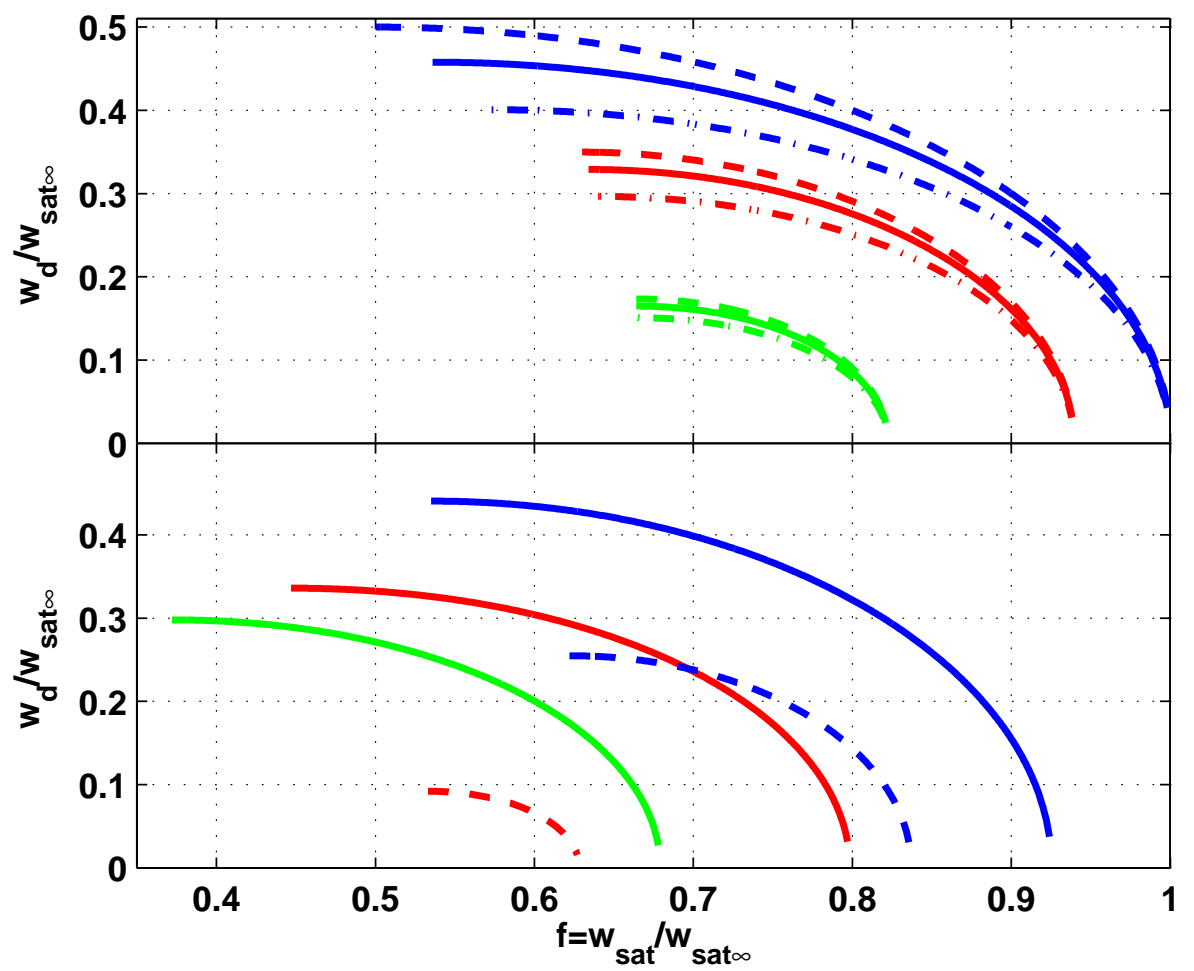

Figure 11. Solution of equation (9), $\hat{w}_{d, e}$ vs $\hat{w}$, with $\bar{a}_{G G J}=0.1$. (a) $f_{\text {bse }}=1$ and $\hat{w}_{m, p o l}=0$ (blue), 0.4 (red) and 0.6 (green). Also shown $\bar{a}_{G G J}=0$ (dashed) and 0.3 (dash-dotted). (b) $f_{b s e}=0.7, \hat{w}_{m, p o l}=0$ (solid lines), 0.4 (dashed lines); and $\hat{w}_{d, i}=0.5$ (blue), 1 (red) and 4 (green).

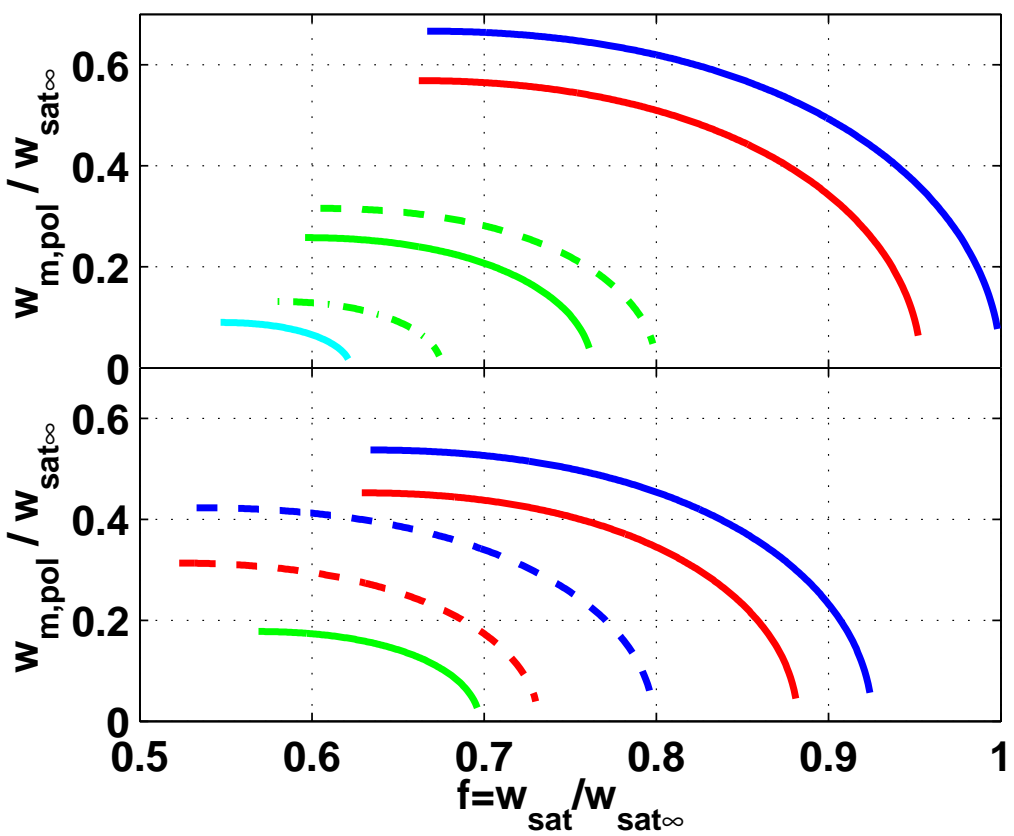

Figure 12. Solution of equation (9), $\hat{w}_{m, p o l}$ vs $\hat{w}$, with $\bar{a}_{G G J}=0.1$. (a) $f_{b s e}=1, \hat{w}_{d, i}=$ 0 and $\hat{w}_{d, e}=0$ (blue), 0.2 (red), 0.4 (green) and 0.45 (cyan). For $\hat{w}_{d, e}=0.4, \bar{a}_{G G J}=0$ (dashed green) and 0.25 (dash-dotted green). (b) $f_{b s e}=0.7, \hat{w}_{d, i}=0.5$ (solid lines), 1 (dashed lines); and $\hat{w}_{d, e}=0$ (blue), 0.2 (red) and 0.4 (green). 

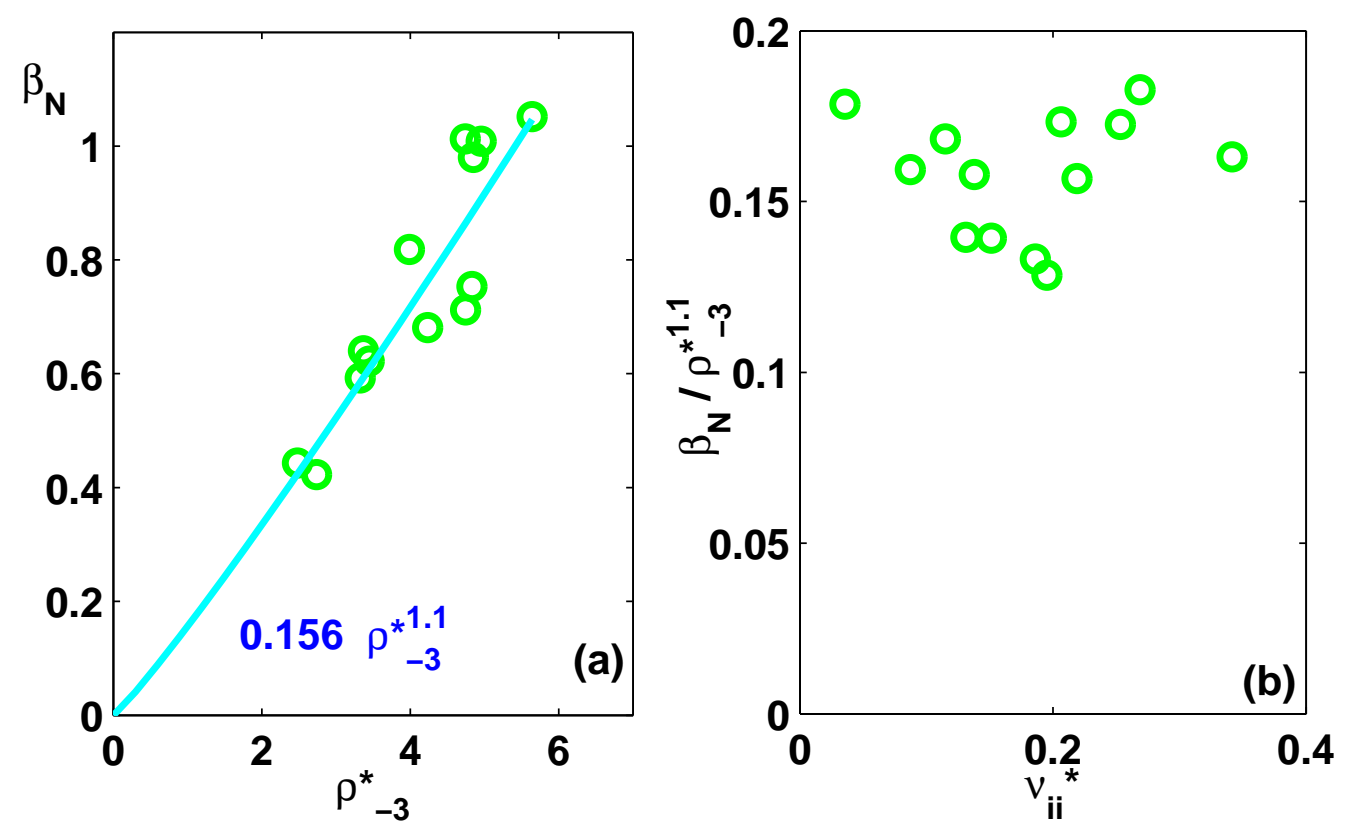

Figure 13. (a) Range of $\beta_{N}$ versus $\rho_{-3}^{*}=10^{3} \rho *$ in the experiments described in this paper, with a linear correlation coefficient $\mathrm{R}=0.92$. Examples of global and local parameters are given in table 1. (b) Assuming the $\rho *$ scaling shown in (a), no dependence on $\nu_{i i} *$ remains, however further experiments at much larger $\nu_{i i} *$ are needed. About half of the discharges have mode stabilisation after the H-L transition, therefore larger uncertainties in the data. Typically those with very slow ramp-down, like \#53634, have the stabilisation before the H-L transition. 